Discrete Comput Geom 31:461-490 (2004)

DOI: $10.1007 / \mathrm{s} 00454-003-2877-\mathrm{x}$

\title{
Classes of Discrete Convexity Properties
}

\author{
Gabriela Cristescu ${ }^{1}$ and Liana Lupşa ${ }^{2}$ \\ ${ }^{1}$ Department of Mathematics and Computer Science, Aurel Vlaicu University of Arad, \\ No. 81 Str. Revolutiei, 310130 Arad, România \\ gcristescu@inext.ro \\ ${ }^{2}$ Faculty of Mathematics and Computer Science, Babeş-Bolyai University, \\ No. 1 Str. Kogălniceanu, 400084 Cluj-Napoca, România \\ llupsa@math.ubbcluj.ro
}

\begin{abstract}
In the general first-level classification of the convexity properties for sets [16], discrete convexities appear in more classes. A second-level classification identifies more subclasses containing discrete convexity properties, which appear as approximations either of classical convexity or of fuzzy convexity. First, we prove that all these convexity concepts are defined by segmental methods. The type of segmental method involved in the construction of discrete convexity determines the subclass to which it belongs. The subclasses containing the convexity properties that have discrete particular cases are also presented.
\end{abstract}

\section{Convexities Defined by Segmental Methods}

The classical framework for convexity is a nonempty set $X$ in which the notion of a straight-line segment is defined. The following definition introduces the notion, which we refer to as classical convexity.

Definition 1.1. A subset $A$ of $X$ is convex if whenever it contains two points, it also contains the straight-line segment joining them.

Therefore, in a convex set, for every two points $x \in A$ and $y \in A$, the straight-line segment joining them, denoted by $\langle x, y\rangle$, is also contained in $A$. In a real linear space, $(X,+, \cdot, \mathbf{R})$, the straight-line segment joining the points $x$ and $y$ is defined by

$$
\langle x, y\rangle=\{z \in X \mid z=t x+(1-t) y, 0 \leq t \leq 1\} .
$$


From this definition we see that the straight-line segment is a set isomorphic to interval $[0,1]$, the isomorphism being $s:[0,1] \rightarrow\langle x, y\rangle$ defined by $s(t)=t x+(1-t) y$. It means that the straight-line segment imitates the real interval $[0,1]$, implying the connectivity of a classically convex set. Throughout the paper $\operatorname{conv}(A)$ is used for the classical convex hull of set $A$ in a linear space, meaning the union of all straight-line segments joining points of $A$.

In this paper we discuss the methods of defining various concepts of a convex set in a discrete context. They are obtained by modifying the straight-line segment such that connectivity is not necessarily implied. We use the term segmental methods to define this type of technique.

The segmental methods may be grouped into several classes, according to the way in which each of them is elaborated:

1. Replacing the linear structure of the space by another type of structure and defining the notion of a straight-line segment.

2. In linear space, choosing a particular subset of the straight-line segment and using it instead of the straight-line segment.

3. In linear space, choosing a certain type of straight-line segment from the set of all straight-line segments.

4. In a space, in which the notion of a straight-line segment is defined, another condition besides that of belonging to the set is supposed to be satisfied by the straight-line segment.

5. In a space, in which the notion of a straight-line segment is defined, points outside the straight-line segment are considered in relation to the given set.

6. Weak segmental case, when either the straight-line segment determined by two points is not unique or it must be situated in a particular context related to the set under discussion.

Methods 1-4 are used to define discrete convex sets. Methods 4-6 usually lead to convexity properties, which either have discrete particular cases or contain discrete sets among the convex sets according to the definition.

The general first-level classification of 100 notions of convexity properties for sets, contained in [16] is based on a definition of a generalized convexity concept that becomes the classifier. This definition is based on the identification of more common elements of all the convexity concepts from the collection. First, classical convexity is a particular case of each of them. Another common point is that all 100 concepts of convexity may be written using some key elements, obtaining a general classifying notion. Using these elements, the set of 100 convexities was divided into 11 classes by the manner in which they may appear in a definition. A second-level classification, according to structural criteria, groups the discrete convexities into subclasses, which are presented in this paper.

The key elements that are involved in the process of the elaboration of a convexity theory for sets are:

- A nonempty set $X$, which is the support set.

- A nonempty set $Y$.

- A nonempty subset $S \subseteq 2^{X}$. 
- An $\mathcal{S}$ family of functions, $s: S \rightarrow 2^{Y}$.

- An $\mathcal{R}$ family of functions, $r: 2^{X} \rightarrow 2^{Y}$.

The elements of the subset $S$ of the set of the parts of $X$ will replace the pairs of points which appear in the definition of classical convexity. The function $s: S \rightarrow 2^{Y}$ will replace the straight-line segment determined by a pair of elements, which appear in the definition of classical convexity. The function $r: 2^{X} \rightarrow 2^{Y}$ will be used to rewrite the condition that every straight-line segment determined by pairs of elements of the set under consideration belong to this set. In the case of classical convexity the $\mathcal{R}$ family is a singleton and its unique element is the identity on $2^{X}$.

Condition $s(C) \subseteq r(A)$ has to be satisfied in every definition of a convexity property of a set $A \subseteq X$, for $C \subseteq A$ and $C \in S$.

All the discrete convexity properties and the convexity properties including discrete particular cases from our collection may be modelled using the above-described elements as segmental convexity.

The discrete convexity properties are grouped in the segmental subclasses of four classes identified in [16], as shown in Table 1.1.

Another type of convexity properties that are of interest from a discrete point of view consists in those convexity concepts having discrete particular cases. They appear in many applications in integer and rational programming.

Therefore, the convexity properties with discrete particular cases are grouped in the segmental subclasses of five classes identified in [16] (see Table 1.2). In what follows we

Table 1.1. Classes of discrete convexity properties.

\begin{tabular}{|c|c|c|c|c|}
\hline No. & $\begin{array}{l}\text { Type of convexity } \\
\text { in the class }\end{array}$ & $\begin{array}{l}\text { Number } \\
\text { of } \\
\text { members }\end{array}$ & $\begin{array}{l}\text { Number of } \\
\text { discrete } \\
\text { convexities }\end{array}$ & $\begin{array}{c}\text { Members } \\
\text { discrete convexities }\end{array}$ \\
\hline 1 & $(S, s)$ convexity & 42 & 2 & $\begin{array}{l}\text { - Discrete convexity in } \\
\mathbf{Z}^{2} \text { [32] } \\
\text { - Discrete convexity in } \\
\mathbf{Z}^{2} \text { [34], [35] }\end{array}$ \\
\hline 2 & $\begin{array}{l}\text { Special partial } \\
((S, s), r) \text { convexity }\end{array}$ & 7 & 4 & $\begin{array}{l}\text { - } \text { Convexity of cellular } \\
\text { blobs [36] } \\
\text { - } \text { Digital convexity in } \\
\mathbf{Z}^{2}[18] \\
\text { - } \\
\mathbf{Z}^{2} \text { [20] } \\
\text { - Fuzzy digital } \\
\text { convexity [17] }\end{array}$ \\
\hline 3 & $\begin{array}{l}(e, a)-((S, \mathcal{S}), r) \\
\text { convexity }\end{array}$ & 21 & 3 & $\begin{array}{l}\text { - } \text { Collinear 3-point } \\
\text { convexity [21] } \\
\text { - Discrete convexity [2], [3] } \\
\text { - Discrete convexity [19] }\end{array}$ \\
\hline 4 & $\begin{array}{l}\text { Special partial } \\
(a, e)-((S, s), \mathcal{R})\end{array}$ & 4 & 2 & $\begin{array}{l}\text { - Fuzzy ortho-convexity [5] } \\
\text { - Fuzzy ortho-concavity [7] }\end{array}$ \\
\hline
\end{tabular}


Table 1.2. Convexity properties having discrete particular cases.

\begin{tabular}{|c|c|c|c|c|}
\hline No. & $\begin{array}{l}\text { Type of convexity } \\
\text { in the class }\end{array}$ & $\begin{array}{l}\text { Number } \\
\text { of } \\
\text { members }\end{array}$ & $\begin{array}{l}\text { Number of } \\
\text { discrete } \\
\text { convexities }\end{array}$ & $\begin{array}{c}\text { Members } \\
\text { discrete convexities }\end{array}$ \\
\hline 1 & $(S, s)$ convexity & 42 & 10 & $\begin{array}{l}\text { - Strong convexity with } \\
\text { respect to a given set [22] } \\
\text { - Integer convexity [24] } \\
\text { - Slack convexity with } \\
\text { respect to a given set [26] } \\
\text { - } k \text {-Strong convexity } \\
\text { with respect to a given } \\
\text { set [27] } \\
\text { - } k \text {-Slack convexity } \\
\text { with respect to a given } \\
\text { set [27] } \\
\text { - } k \text {-Strong convexity } \\
\text { with respect to } g \text { and } M \text { [1] } \\
\text { - } k \text {-Slack convexity } \\
\text { with respect to } g \text { and } M[1] \\
\text { - Strong convexity } \\
\text { with respect to } g \text { and } M \text { [1] } \\
\text { - Slack convexity } \\
\text { with respect to } g \text { and } M \\
\text { set [1] } \\
\text { - Induced seg-convexity } \\
\text { with respect to } p, f \text { and } M[1]\end{array}$ \\
\hline 2 & $((S, s), r)$ convexity & 13 & 5 & $\begin{array}{l}\text { - Convexity with } \\
\text { respect to a behaviour [12] } \\
\text { - } \alpha \text {-convexity [11] } \\
\text { - Slack convexity with } \\
\text { respect to a set and } \\
\text { two behaviours [9] } \\
\text { - Slack }(\alpha, \delta) \text {-convexity } \\
\text { with respect to a } \\
\text { network [8] } \\
\text { - Induced } k \text {-strong } \\
\text { convexity with respect } \\
\text { to } p, f \text { and } M[1]\end{array}$ \\
\hline 3 & $\begin{array}{l}\text { Special partial } \\
((S, s), r) \text { convexity }\end{array}$ & 7 & 1 & $\begin{array}{l}\text { - Induced strict strong } \\
\text { convexity with respect } \\
\text { to } p, f \text { and } M \text { [1] }\end{array}$ \\
\hline 4 & $\begin{array}{l}(e, a)-((S, \mathcal{S}), r) \\
\text { convexity }\end{array}$ & 21 & 2 & $\begin{array}{l}\text { - Strong convexity with } \\
\text { respect to a set and } \\
\text { two behaviours [9] } \\
\text { - Strong }(\alpha, \delta) \text {-convexity } \\
\text { with respect to a } \\
\text { network [8] }\end{array}$ \\
\hline 5 & $\begin{array}{l}\text { Special partial } \\
(a, e)-((S, \mathcal{S}), \mathcal{R}) \\
\text { convexity }\end{array}$ & 4 & 1 & $\begin{array}{l}\text { - Induced strong } \\
\text { convexity with respect } \\
\text { to } p, f \text { and } M \text { [1] }\end{array}$ \\
\hline
\end{tabular}


present both the subclasses of discrete convexity properties and those including discrete particular cases, identifying some relations between them.

\section{Discrete $(S, s)$ Convexity Concepts}

All the discrete convexity properties of the type described in this class are obtained by replacing the straight-line segment with one of its subsets identified by the condition of having integer coordinates. $(S, s)$ convexity is obtained supposing that $X=Y$, the $\mathcal{S}$ family is a singleton, $\mathcal{S}=\{s\}$ with $s: S \rightarrow 2^{X}$, and the $\mathcal{R}$ family is also a singleton, containing only the identity on $2^{X}, \mathcal{R}=\left\{1_{2^{X}}\right\}$.

Definition 2.1. A set $A \subseteq X$ is said to be $(S, s)$ convex if $s(C) \subseteq A$, for each $C \subseteq A$, with $C \in S$.

\section{1. $(S, s)$ Discrete Convexities}

The following examples present all the $(S, s)$ discrete convexities, i.e. the $(S, s)$ convexity properties of sets in case $X=\mathbf{Z}^{2}$.

Example 2.1. Discrete convexity in $\mathbf{Z}^{2}$ [32].

In $\mathbf{Z}^{2}$ the distance

$$
c(x, y)=\max \left\{\left|x_{1}-y_{1}\right|,\left|x_{2}-y_{2}\right|\right\}, \quad x=\left(x_{1}, x_{2}\right) \in \mathbf{Z}^{2}, \quad y=\left(y_{1}, y_{2}\right) \in \mathbf{Z}^{2},
$$

is used. The connectivity by means of arcs induced by this distance is considered and is called, as usual, 8-connectivity.

Definition 2.2. The 8-connected component $A$ in $\mathbf{Z}^{2}$ is said to be discrete convex if there is not a triplet $\left(P_{1}, P_{2}, P_{3}\right)$ of collinear points, with $P_{2}$ situated between $P_{1}$ and $P_{3}$, such that $P_{1}$ and $P_{3}$ are situated in $A$ and $P_{2}$ belongs to the complement of $A$.

It means that each triplet of collinear points $\left(P_{1}, P_{2}, P_{3}\right)$ with $P_{1}$ and $P_{3}$ situated in $A$ has the property that $P_{2}$ lies in $A$.

We take $X=Y=\mathbf{Z}^{2}, S=\left\{\left\{P_{1}, P_{2}\right\} \mid P_{1} \in \mathbf{Z}^{2}, P_{2} \in \mathbf{Z}^{2}\right\}, s: S \rightarrow 2^{X}$ defined by

$$
s\left(\left\{P_{1}, P_{2}\right\}\right)=\left\{P \mid P \in \mathbf{Z}^{2},\left\{P_{1}, P, P_{2}\right\} \text { collinear, } P \text { between } P_{1} \text { and } P_{2}\right\},
$$

and $r=1_{2^{x}}$.

Theorem 2.1. An 8-connected component $A \subseteq \mathbf{R}^{2}$ is discrete convex if and only if $A$ is $(S, s)$ convex.

Proof. We suppose that $A$ is discrete convex. Then each triplet of collinear points ( $P_{1}$, $\left.P_{2}, P_{3}\right)$ with $P_{1}$ and $P_{3}$ situated in $A$ has the property that $P_{2}$ lies in $A$. Therefore, for 
each subset $\left\{P_{1}, P_{3}\right\} \in S$, with $\left\{P_{1}, P_{3}\right\} \subseteq A$, one has $s\left(\left\{P_{1}, P_{3}\right\}\right) \subseteq r(A)$. It means that $A$ is $(S, s)$ convex.

Conversely, if $A$ is $(S, s)$ convex, then for each $\left\{P_{1}, P_{3}\right\} \in S$, with $\left\{P_{1}, P_{3}\right\} \subseteq A$, $s\left(\left\{P_{1}, P_{3}\right\}\right) \subseteq r(A)$ occurs. It means that each point $P_{2}$ collinear with $P_{1}$ and $P_{3}$ and situated between them, lies in $A=r(A)$. Therefore, there is not a triplet $\left(P_{1}, P_{2}, P_{3}\right)$ of collinear points, with $P_{2}$ situated between $P_{1}$ and $P_{3}$, such that $P_{1}$ and $P_{3}$ are situated in $A$ and $P_{2}$ belongs to the complement of $A$. It means that $A$ is discrete convex.

Example 2.2. Discrete convexity [34] (see also [35]).

Recall that if $x=(i, j) \in \mathbf{Z}^{2}$, then the 8-neighbourhood of point $x$ is the set

$$
\begin{aligned}
V_{8}(x)= & \{x,(i-1, j-1),(i-1, j),(i-1, j+1),(i, j-1),(i, j+1), \\
& (i+1, j-1),(i+1, j),(i+1, j+1)\} .
\end{aligned}
$$

Each point belonging to $V_{8}(x)$ is said to be an 8-neighbour of $x$.

According to Rosenfeld and Kak,

Definition 2.3. A set $A \subseteq \mathbf{Z}^{2}$ is discrete convex if every straight-line intersects $A$ at most once, meaning only in a succession of 8-neighbour points.

It is obvious that this definition is equivalent to that by Minsky and Papert, therefore they belong to the same class. This concept of discrete convexity generated investigations into the situation when the above-mentioned intersection property holds only for straightlines with particular directions, leading to directional convexity.

\section{2. $(S, s)$ Convexities with Discrete Particular Cases}

All the convexity properties from this subclass have discrete particular cases. Also, the convexities defined by these definitions in nondiscrete spaces do not imply connectivity in the topology of the space, including discrete sets among the convex sets.

Example 2.3. Strong convex sets with respect to $M$ [24].

$(V,+, \cdot)$ is supposed to be a real linear space and $M$ is a nonempty subset of $V$.

Definition 2.4. A subset $A \subseteq V$ is called strongly convex with respect to $M$ if $A=\emptyset$ or if $M \cap \operatorname{conv}(A) \subseteq A$.

For $X=Y=V, S=2^{V}$ and the function $s: S \rightarrow 2^{V}$ defined by $s(B)=M \cap$ $\operatorname{conv}(B)$, for all $B \in S$, one can easily prove the following:

Theorem 2.2. A set $A \subseteq V$ is strongly convex with respect to $M$ if and only if $A$ is $(S, s)$ convex. 
Examples of the strong convexity with respect to a given set are the directional convexities from [33]. Also, the conic approach to convexity from [23] and [38] are at the origin of the approach to convexity from Definition 2.4. Further properties and applications in integer and rational programming are presented in papers [25]-[31].

The following example will prove that strong convexity with respect to a set $M$ is a concept having discrete particular cases.

Example 2.4. Integer convexity [24].

Definition 2.5. A subset $X \subseteq \mathbf{Z}^{n}$ is said to be an integer convex set if $X$ is strongly convex with respect to $\mathbf{Z}^{\mathrm{n}}$.

The following characterization proves that the integer convexity includes the discrete convexity of Rosenfeld and $\operatorname{Kak}[34]$. Indeed, set $A=\{(0,0),(0,1),(1,0),(1,1),(1,2)\}$ is integer convex without being discrete convex according to Definition 2.3. It is easy to see that the intersection between set $A$ and the straight line $y=2 x$ is the set $\{(0,0),(1,2)\}$, which contains two points that are not 8-neighbours (Fig. 1). If the concept of a straight line is replaced by a digital straight line, according to the description from [4], then it is possible to find conditions for an integer convex set to be discrete convex as well (Fig. 2).

Theorem 2.3. A set $X \subseteq \mathbf{Z}^{n}$ is integer convex if and only if for any two elements $x=\left(x_{1}, \ldots, x_{n}\right)$ and $y=\left(y_{1}, \ldots, y_{n}\right)$ of $X$ and for any natural number $r, r$ is a common divisor of numbers $\left|x_{j}-y_{j}\right|, j \in\{1, \ldots, n\}$, we have

$$
\frac{s}{r} x+\left(1-\frac{s}{r}\right) y \in X, \quad \text { for each } s \in\{0,1, \ldots, r\} .
$$

Proof. We suppose that $r$ is a common divisor of numbers $\left|x_{j}-y_{j}\right|, j \in\{1, \ldots, n\}$, and $s \in\{0,1, \ldots, r\}$. Therefore, for each $j \in\{1, \ldots, n\}$ there is a number $u_{j} \in \mathbf{Z}$ such

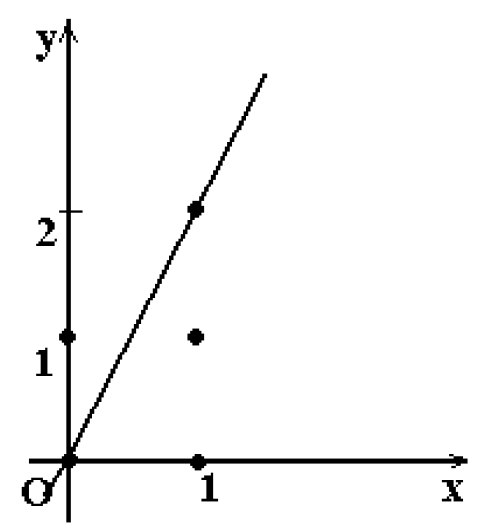

Fig. 1. An integer convex set which is not discrete convex. 


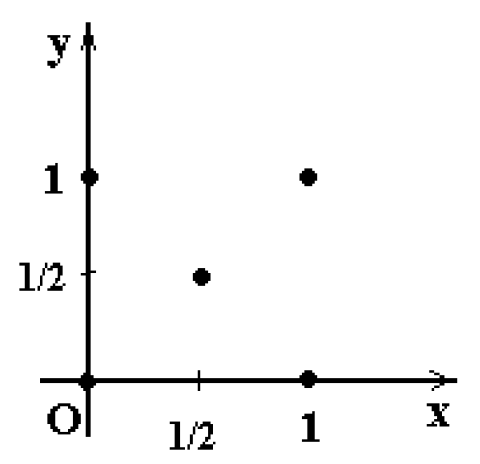

Fig. 2. An integer convex set which is discrete convex.

that $x_{j}-y_{j}=r u_{j}$. We obtained a vector $u=\left(u_{1}, \ldots, u_{n}\right) \in \mathbf{Z}^{n}$. As $x \in \mathbf{Z}^{n}, y \in \mathbf{Z}^{n}$, it follows that

$$
\frac{s}{r} x+\left(1-\frac{s}{r}\right) y=y+\frac{s}{r}(x-y)=y+s u \in \mathbf{Z}^{n},
$$

and because $X$ is an integer convex set, we get that (1) is true.

Conversely, supposing that for each pair of points $x, y \in X$, for each number $r$, which is a common divisor of numbers $\left|x_{j}-y_{j}\right|, j \in\{1, \ldots, n\}$, it follows that $p=$ $(s / r) x+(1-s / r) y \in X$, for each integer $s \in\{0,1, \ldots, r\}$. Let $x, y$ be two elements of $X$ and let $t \in$ ]0, 1[. Suppose that $z=t x+(1-t) y \in \mathbf{Z}^{n}$. In order to prove the integer convexity of set $X$ we must show that $z \in X$. Since $z=t x+(1-t) y \in \mathbf{Z}^{n}$ and $x-y \in X \subseteq \mathbf{Z}^{n}$, it follows that $t(x-y) \in \mathbf{Z}^{n}$, which implies that $t$ is a rational number. As $t \in] 0,1$, it follows that there are two numbers $r, s \in \mathbf{N}$, such that $t=s / r$ and fraction $s / r$ is irreducible. Number $r$ is a common divisor of all the components of vector $\left(\left|x_{1}-y_{1}\right|, \ldots,\left|x_{n}-y_{n}\right|\right)$ because $t(x-y) \in \mathbf{Z}^{n}$ and $s / r$ is irreducible. On the other hand, $t=s / r \in] 0,1$ [ irreducible implies that $s \in\{1, \ldots, r-1\}$. In this case, according to our hypothesis, $z=(s / r) x+(1-s / r) y \in X$, meaning that $X$ is an integer convex set.

The intersection property contained in the definition of [34] is now obvious. It is also obvious, according to the previous example, that integer convexity is a property of $(S, s)$ convexity.

Example 2.5. Slack convex sets with respect to $M$ [24].

Let $(V,+, \cdot)$ be a real linear space and let $M$ be a nonempty subset of $V$.

Definition 2.6. A subset $A \subseteq V$ is called slackly convex with respect to $M$ if $X=\emptyset$ or if $M \cap \operatorname{conv}(M \cap A) \subseteq A$.

We choose $Y=X, S=2^{X}$ and consider function $s: S \rightarrow 2^{X}$ defined by $s(B)=$ $M \cap \operatorname{conv}(M \cap B)$, for all $B \in S$. As usual, we can prove the following result. 
Theorem 2.4. A set $A \subseteq V$ is slackly convex with respect to $M$ if and only if $A$ is $(S, s)$ convex.

There are discrete sets that are slackly convex with respect to $M$, without having the strong property. For example, let $X=\left\{(0,2),\left(\frac{1}{2}, 0\right),(1,3),(2,3)\right\}$. Since $\mathbf{Z}^{2} \cap$ $\operatorname{conv}(X)=\{(0,2),(1,1),(1,2),(1,3),(2,3)\} \not \subset X$, set $X$ is not strongly convex with respect to $\mathbf{Z}^{2}$. However, set $X$ is slackly convex with respect to $\mathbf{Z}^{2}$ because we have $\mathbf{Z}^{2} \cap \operatorname{conv}\left(\mathbf{Z}^{2} \cap X\right)=\{(0,2),(1,3),(2,3)\} \subseteq X$.

Example 2.6. Strong $k$-convexity with respect to a given set [24].

Let $(V,+, \cdot)$ be a real linear space. In the following we denote a nonempty subset of $V$ by $M$. Let $k$ be a natural number, $k>0$.

Definition 2.7. A subset $A \subseteq V$ is called strongly $k$-convex with respect to $M$ if $A=\emptyset$ or if for each system $x_{1}, \ldots, x_{k} \in A$ and all real numbers $t_{1}, \ldots, t_{k}$ satisfying the conditions $t_{1} \geq 0, \ldots, t_{k} \geq 0, t_{1}+\cdots+t_{k}=1$ and $t_{1} x_{1}+\cdots+t_{k} x_{k} \in M$, we have $t_{1} x_{1}+\cdots+t_{k} x_{k} \in A$.

If we take $Y=X=V, S=\left\{\left\{x_{1}, x_{2}, \ldots, x_{k}\right\} \mid x_{1}, x_{2}, \ldots, x_{k} \in X\right\}$, function $s: S \rightarrow$ $2^{X}$ is defined by $s(B)=g(B) \cap M$, for all $B \in S$, then we obtain that the $k$-strong convexity with respect to a given set is an $(S, s)$ convexity.

Examples 2.3 and 2.4 are particular cases of the $k$-strong convexity with respect to a given set. It is easy to see that if a set $A \subseteq X$ is $k$-strongly convex with respect to $M$, where $k$ is a natural number, then $A$ is also $m$-strongly convex with respect to $M$ for each natural number $m, m<k$. The converse is not always true. Let $A=\{(-1,0),(0,2),(1,1)\}$. Set $A$ is 2 -strongly convex with respect to $\mathbf{Z}^{2}$, but it is not 3 -strongly convex with respect to $\mathbf{Z}^{2}$. Obviously, set $A$ is not strongly convex with respect to $\mathbf{Z}^{2}$, i.e. integer convex.

Also, we remark that if $\operatorname{dim} V=k$, then a subset $A$ of $V$ is strongly convex with respect to $M$ if and only if it is $k$-strongly convex with respect to $M$.

Example 2.7. $k$-Strong convexity with respect to $g$ and $M$ [1].

Let $X$ and $M$ be two arbitrary nonempty sets and let $g$ be a convex hull operator on $X$. Let $k$ be a natural number, $k \geq 2$.

Definition 2.8. A subset $A \subseteq X$ is called $k$-strongly convex with respect to $g$ and $M$ if for any $a_{1}, \ldots, a_{k}$ elements of $A$ we have $g\left(a_{1}, \ldots, a_{k}\right) \cap M \subseteq A$.

We choose $Y=X, S=\left\{\left\{x_{1}, x_{2}, \ldots, x_{k}\right\} \mid x_{1}, x_{2}, \ldots, x_{k} \in X\right\}, s: S \rightarrow 2^{X}$ defined by $s(B)=g(B) \cap M$ for all $B \in S$.

Theorem 2.5. A set $A \subseteq X$ is $k$-strongly convex with respect to $g$ and $M$ if and only if $A$ is $(S, s)$ convex. 
Obviously, if $V$ is a linear space, $M$ is a nonempty subset of $V$ and $k$ is a natural number, $k \geq 2$, then $k$-strong convexity with respect to $M$ is a particular case of $k$-strong convexity with respect to $g$ and $M$, where $g$ is takes the classical convex hull.

A nice example of 2-strongly convexity with respect to $g$ and $M$ is the following. Let $M=\{2 p \mid p$ odd number $\}$ and let $g: 2^{\mathbf{N}^{*}} \rightarrow 2^{\mathbf{N}^{*}}$ be a map with

$$
g(\emptyset)=\emptyset, \quad g\left(\mathbf{N}^{*}\right)=\mathbf{N}^{*}
$$

and

$$
g(A)=\left\{u \in \mathbf{N}^{*} \mid \text { g.c.d. }(A) \leq u \leq \text { l.e.m. }(A)\right\} \backslash\{4\},
$$

where g.c.d.( $A$ ) denote the greatest common divisor of the elements of $A$ and l.e.m. $(a, b)$ the least common multiple of these elements; we take l.e.m. $(a, b)=+\infty$, if $\sup (A)=$ $\infty$. Set $A=\{2,3,6\}$ is 2 -strongly convex with respect to $g$ and $M$, but, because $4 \in M \cap g(\{3,6\})$, it is not 2-strongly convex with respect to $M$.

Example 2.8. Strong convexity with respect to $g$ and $M[1]$.

Let $X$ and $M$ be two arbitrary nonempty sets and let $g$ be a convex hull operator on $X$ (i.e. a mapping $g: 2^{X} \rightarrow 2^{X}$ satisfying the conditions $A \subseteq g(A), g(g(A))=g(A)$, $g(B) \subseteq g(A)$, for any subsets $A, B \in 2^{X}, B \subseteq A$ ).

Definition 2.9. A subset $A \subseteq X$ is called strongly convex with respect to $g$ and $M$ if $g(A) \cap M \subseteq A$.

We choose $Y=X, S=2^{X}, s: S \rightarrow 2^{X}$ defined by $s(B)=g(B) \cap M$, for all $B \in S$. As usual, we can prove the following result.

Theorem 2.6. A set $A \subseteq X$ is strongly convex with respect to $g$ and $M$ if and only if $A$ is $(S, s)$ convex.

Example 2.3 presents a particular case of strong convexity with respect to $g$ and $M$. Because $g$ is a convex hull operator on $X$, if a set $A \subseteq X$ is strongly convex with respect to $g$ and $M$, then $A$ is $k$-strongly convex with respect to $g$ and $M$, for each natural number $k$..

Example 2.9. Slack $k$-convexity with respect to $g$ and $M$ [1].

Let $X$ and $M$ be two arbitrary nonempty sets and let $g$ be a convex hull operator on $X$. Let $k$ be a natural number, $k \geq 2$.

Definition 2.10. A subset $A \subseteq X$ is called slackly $k$-convex with respect to $g$ and $M$ if for any $a_{1}, \ldots, a_{k}$ elements of $A$ we have $M \cap g\left(\left\{a_{1}, \ldots, a_{k}\right\} \cap M\right) \subseteq A$.

If we choose $Y=X, S=\left\{\left\{x_{1}, x_{2}, \ldots, x_{k}\right\} \mid x_{1}, x_{2}, \ldots, x_{k} \in X\right\}, s: S \rightarrow 2^{X}$ defined by $s(B)=M \cap g(B \cap M)$ for all $B \in S$, then we can prove that a set $A \subseteq X$ is slackly $k$-convex with respect to $g$ and $M$ if and only if $A$ is $(S, s)$ convex. 
We remark that if a set $A \subseteq X$ is strongly $k$-convex with respect to $g$ and $M$, then it is also slackly $k$-convex with respect to $g$ and $M$.

Example 2.10. Slack convexity with respect to $g$ and $M[1]$.

Let $X$ and $M$ be two arbitrary nonempty sets and let $g$ be a convex hull operator on $X$.

Definition 2.11. A subset $A \subseteq X$ is called slackly convex with respect to $g$ and $M$ if $M \cap g(A \cap M) \subseteq A$.

For $Y=X, S=2^{X}$ and the function $s: S \rightarrow 2^{X}$ defined by $s(B)=M \cap g(B \cap M)$ for all $B \in S$, it is easy to prove that a set $A \subseteq X$ is slackly convex with respect to $g$ and $M$ if and only if $A$ is $(S, s)$ convex.

Example 2.11. Slack $n$-convex sets with respect to $M$ [24].

Let $(V,+, \cdot)$ be a real linear space and let $M$ be a nonempty subset of $V$. Let $n$ be a natural number, $n>0$.

Definition 2.12. A subset $A \subseteq V$ is called slackly $n$-convex with respect to $M$ if $A=\emptyset$ or if for all system $x_{1}, \ldots, x_{n} \in M \cap A$ and all real numbers $t_{1}, \ldots, t_{n}$ satisfying conditions $t_{1} \geq 0, \ldots, t_{n} \geq 0, t_{1}+\cdots+t_{n}=1$, and $t_{1} x_{1}+\cdots+t_{n} x_{n} \in M$, we have $t_{1} x^{1}+\cdots+t_{n} x^{n} \in A$.

The property of slack $n$-convexity with respect to a given set $M$ is a particular case of $n$-slack convexity with respect to $g$ and $M$. Therefore, it is also an $(S, s)$ convexity.

Example 2.12. Induced seg-convexity with respect to $p, f$ and $M$ [1].

Let $X, T$ be two arbitrary sets, and let $M$ be a nonempty subset of $T$. Consider the functions $f: X \rightarrow T$ and $p: 2^{T} \rightarrow 2^{T}$. For every point $x, y \in M$, we define the set

$$
\begin{aligned}
\langle x, y\rangle_{p, f, M} & =f^{-1}(p(\{f(x), f(y)\}) \cap M) \\
& =\{z \in X \mid f(z) \in p(\{f(x), f(y)\}) \cap M\} .
\end{aligned}
$$

Definition 2.13. A subset $A \subseteq X$ is called induced seg-convex with respect to $p, f$ and $M$ if $\langle x, y\rangle_{p, f, M} \subseteq A$, for any $x, y \in A$.

As usual, taking $Y=X, S=\{\{x, y\} \mid x \in X, y \in X\}$ and the function $s: S \rightarrow 2^{X}$ defined by

$$
s(\{x, y\})=\langle x, y\rangle_{p, f, M}=f^{-1}(p(\{f(x), f(y)\}) \cap M)
$$

for every $\{x, y\} \in S$, we obtain that the property of induced seg-convexity with respect to $p, f$ and $M$ is an $(S, s)$ convexity. 
There are particular discrete cases of this property of convexity. For example, let $X=\{-1,1,2\}, Y=\{-1,0,1,2\}$ and let $f: X \rightarrow Y$ given by $f(-1)=1, f(1)=1$, $f(2)=2$. Let $p: 2^{Y} \rightarrow 2^{Y}$ given by

$$
\begin{gathered}
p(\emptyset)=\emptyset, \quad p(\{-1\})=\{-1\}, \quad p(\{0\})=\{0\}, \quad p(\{1\})=\{0,1\}, \\
p(\{2\})=\{0,1,2\}, \quad p(\{-1,0\})=\{0\}, \quad p(\{-1,1\})=\{0,1\}, \\
p(\{-1,2\})=\{0,1,2\}, \quad p(\{0,1\})=\{0,1\}, \quad p(\{0,2\})=\{0,1,2\}, \\
p(\{1,2\})=\{1,2\}, \quad p(\{-1,0,1\})=\{0,1\}, \quad p(\{-1,0,2\})=\{0,2\}, \\
p(\{-1,1,2\})=\{1,2\}, \quad p(\{0,1,2\})=\{0,1,2\}, \\
p(\{-1,0,1,2\})=\{-1,0,1,2\} .
\end{gathered}
$$

Let $M=Y$ and $A=\{-1,1\}$. Since $\langle-1,1\rangle_{s, f, M}=\{z \in X \mid f(z) \in\{0,1\}\}=$ $\{-1,1\} \subseteq A$, then set $A$ is seg-convex with respect to $p, f$ and $M$.

\section{3. $((S, s), r)$ Convexity Properties Having Discrete Particular Cases}

The convexity properties from this class are obtained by a segmental method with two variants: by adding to a straight-line segment some points from its neighbourhood, sometimes choosing them by looking into the given set through a network (as in the case of a digitization process). This class contains only convexity properties having discrete particular cases.

Supposing that the $\mathcal{S}$ family is a singleton, $\mathcal{S}=\{s\}$ with $s: S \rightarrow 2^{Y}$, and the $\mathcal{R}$ family is also a singleton, $\mathcal{R}=\{r\}$, with $r: 2^{X} \rightarrow 2^{Y}$, we define $((S, s), r)$ convexity as follows:

Definition 3.1. A set $A \subseteq X$ is said to be $((S, s), r)$ convex if $s(C) \subseteq r(A)$ for each $C \subseteq A$ with $C \in S$.

The framework for the convexity properties having discrete particular cases is, generally, provided by $X=\mathbf{Z}^{2}$ or the case when $X$ is a discrete network over a totally bounded metric space.

Example 3.1. $\alpha$-convex sets [11].

Let $(X, d)$ be a metric space together with the notion of a straight-line segment (not necessarily the metric segment) joining every pair $(x, y)$ of points of $X$, denoted by $\langle x, y\rangle$. We choose a real number $\alpha \geq 0$.

Definition 3.2. Set $A \subset X$ is said to be $\alpha$-convex if it is empty or if for each two points $x \in A$ and $y \in A$ each point $t \in\langle x, y\rangle$ satisfies the condition $d(t, A) \leq \alpha$ (Fig. 3).

We take $Y=X, S=\{\{x, y\} \mid x \in X, y \in X\}$ and define two functions $s: S \rightarrow 2^{X}$ and $r: 2^{X} \rightarrow 2^{X}$ by $s(\{x, y\})=\langle x, y\rangle$, for $\{x, y\} \in S, r(C)=\{t \in X \mid d(t, C) \leq \alpha\}$, for $C \subseteq X$. 


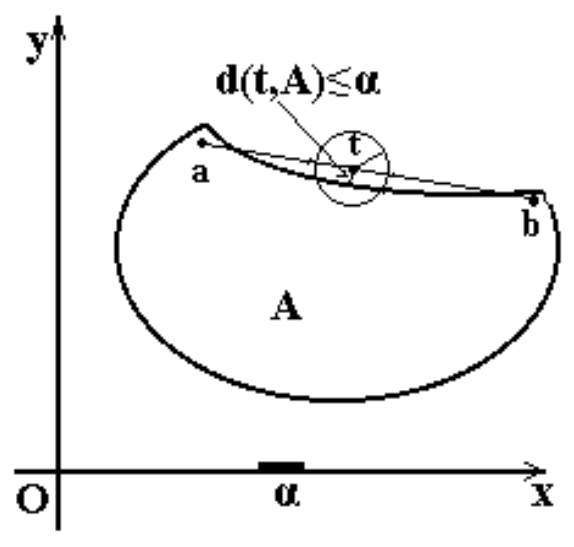

Fig. 3. Set $A$ is $\alpha$-convex.

Theorem 3.1. A set $A \subseteq X$ is $\alpha$-convex if and only if $A$ is $((S, s), r)$ convex.

Proof. Set $A$ is supposed to be $\alpha$-convex. This means that for every $x, y \in A$ and $t \in\langle x, y\rangle$ the inequality $d(t, A) \leq \alpha$ occurs. This is equivalent to the fact that for every $\{x, y\} \in S$ with $x, y \in A$, one has $s(\{x, y\})=\langle x, y\rangle \subseteq\{t \in X \mid d(t, A) \leq \alpha\}=r(A)$. Therefore, $A$ is also $((S, s), r)$ convex.

Conversely, let $A$ be $((S, s), r)$ convex. Let $x, y \in A$. Then $\{x, y\} \in S$. Therefore, $\langle x, y\rangle=s(\{x, y\}) \subseteq r(A)=\{t \in X \mid d(t, A) \leq \alpha\}$, or $\langle x, y\rangle \subseteq\{t \in X \mid d(t, A) \leq \alpha\}$. Hence $A$ is $\alpha$-convex.

There are discrete sets that are $\alpha$-convex. For example, if $X \subset \mathbf{R}^{2}$, together with the Euclidean distance, such that the set

$$
A=([0,1] \backslash \mathbf{Q}) \times([0,1] \backslash \mathbf{Q}) \subset X,
$$

where $\mathbf{Q}$ is the set of all the rational numbers, then the set $A$ is $\alpha$-convex, for all $\alpha \geq 0$. Also, the set $B=G^{2}$, where $G \subset[0,1]$ is the Cantor set in $X=\mathbf{R}^{2}$, is $\frac{1}{2}$-convex.

Remark 3.1. We remark that if the set $A \subset \mathbf{Z}^{2}$ is $\alpha$-convex, with

$$
\alpha<\inf \left\{d(u, v) \mid u, v \in \mathbf{Z}^{2}\right\},
$$

and the straight-line segment is given by $\langle x, y\rangle=\{\lambda x+(1-\lambda y) \mid \lambda \in[0,1]\}$, then $A$ is integer convex.

Indeed, we suppose that $A$ is not integer convex. Then there are two points $x \in A$ and $y \in A$ for which there is a point $t \in\left(\langle x, y\rangle \cap \mathbf{Z}^{2}\right) \backslash A$. As $A \subseteq \mathbf{Z}^{2}$, for point $t$ we have

$$
d(t, A) \geq \inf \left\{d(t, z) \mid z \in \mathbf{Z}^{2}\right\}>\alpha,
$$

which is a contradiction with the hypothesis of $\alpha$-convexity of $A$ (Fig. 4). 


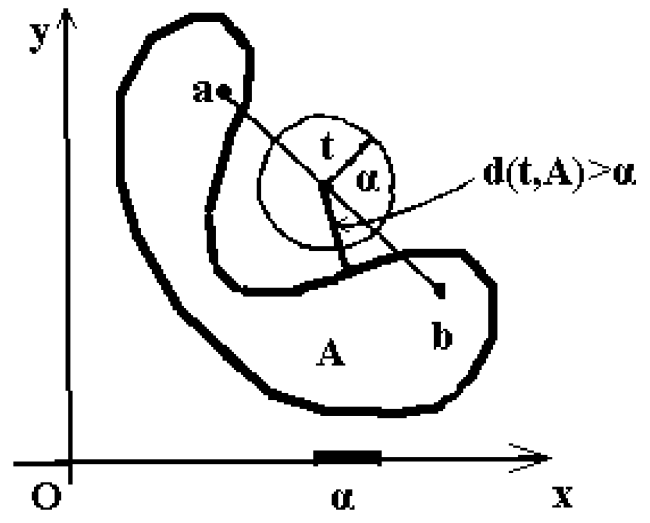

Fig. 4. Set $A$ is not $\alpha$-convex.

The converse is not true, i.e. if $A \subseteq \mathbf{R}^{2}$ is integer convex, then it is not necessary to exist a nonnegative real number $\alpha$, satisfying (3), such that set $A$ is $\alpha$-convex. For example, let $A=\{(1,1),(3,2)\}$ and let $d(x, y)$ denote the Euclidean distance among $x$ and $y$. Set $A$ is integer convex, but it is not 1 -convex (we have $u=\left(2, \frac{3}{2}\right) \in\langle(1,1),(3,2)\rangle$ and $d(u, A)=\sqrt{5} / 2>1)$. Moreover, set $A=\{(1,1),(3,2)\}$ is integer convex without being $\alpha$-convex, with $\alpha<1$. We remark that if $A \subseteq \mathbf{R}^{2}$, but $A \nsubseteq \mathbf{Z}^{2}$, then the conclusion of Remark 3.1 is not always true.

Indeed, let

$$
A=\left\{(x, y) \in \mathbf{R}^{2} \mid 0 \leq x \leq 2,0 \leq y \leq \frac{1}{2} x^{2}\right\} .
$$

Set $A$ is $\frac{1}{2}$-convex, but it is not integer convex because $(1,1)=\frac{1}{2}(0,0)+\frac{1}{2}(2,2)$, but $(1,1) \notin \mathbf{Z}^{2}$.

Example 3.2. Convexity with respect to a behaviour [12].

The notion of behaviour provides us with an important tool to build a theory of generalized convexity (see [8] and [10]-[15]). For a presentation of this notion let $A$ and $B$ be two nonempty sets, let $\mathcal{T}$ be a collection of transformations $T: A \rightarrow B$, let $D$ be a nonempty part of $B$ and let $a \in A$. If $T(a) \in D$, then the pair $(D, T)$ is said to be $a$ strict behaviour of the element a. If $T(a) \in D$ for every $T \in \mathcal{T}$, then the pair $(D, \mathcal{T})$ is said to be a strong behaviour of the element a. If there is a transformation $T \in \mathcal{T}$ such that $T(a) \in D$, then the pair $(D, \mathcal{T})$ is said to be a weak behaviour of the element $a$. If there is an element $a \in A$ such that $T(a) \in D$ (or for every $T \in \mathcal{T}, T(a) \in D$, or there is a $T \in \mathcal{T}$ having the property that $T(a) \in D)$, then the pair $(D, T)$ is said to define a behaviour in $A$ by means of $B$. Then

$$
\begin{aligned}
\operatorname{Comp}(A, B)= & \{(D, T) \mid D \subseteq B, T: A \rightarrow B\} \\
\bigcup & \{s-(D, \mathcal{T}) \mid D \subseteq B, \mathcal{T} \subseteq\{f \mid f: A \rightarrow B\}\} \\
& \bigcup\{w-(D, \mathcal{T}) \mid D \subseteq B, \mathcal{T} \subset\{f \mid f: A \rightarrow B\}\}
\end{aligned}
$$


is called the set of behaviours of $A$ by means of $B$. The elements of the set $\operatorname{Comp}(A, B)$ will be denoted either by $C=(D, T)$ or $C=\mathrm{s}-(D, \mathcal{T})$ or $C=\mathrm{w}-(D, \mathcal{T})$.

In the nonempty set $X$ the notion of a straight-line segment, $\langle x, y\rangle$, for $x, y \in X$, is defined. For the nonempty set $B$ and its nonempty subset $D \subset B$, the behaviour (strict, weak, or strong) $C=(D, \mathcal{T})$ is defined in $X \times 2^{X}$, with $T \in \mathcal{T} \subset\left\{T \mid T: X \times 2^{X} \rightarrow B\right\}$.

Definition 3.3. Set $A \subset X$ is called convex with respect to behaviour $C$ if it is empty or if for every two points $x, y \in A$ and for every $t \in\langle x, y\rangle$, the condition $(t, A) \in C\left(X \times 2^{X}\right)$ holds.

The convexity presented in the previous example is a particular case of convexity with respect to a behaviour. Indeed, we can take $T: X \times 2^{X} \rightarrow \mathbf{R}$ defined by $T(t, A)=$ $d(t, A)=\inf \{d(t, x) \mid x \in A\}$, and the behaviour $C=([0, \alpha], T)$ in $X \times 2^{X}$. As above, we take $Y=X, S=\{\{x, y\} \mid x \in X, y \in X\}$ and we define functions $s: S \rightarrow 2^{X}$ and $r: 2^{X} \rightarrow 2^{X}$ by $s(\{x, y\})=\langle x, y\rangle$, for $\{x, y\} \in S$ and $r(Z)=\{t \in X \mid(t, Z) \in$ $\left.C\left(X \times 2^{X}\right)\right\}$, for $Z \subseteq X$. Then a set $A \subseteq X$ is convex with respect to the behaviour $C$ if and only if $A$ is $((S, s), r)$ convex.

All the convexity properties from Section 2, except those from Examples 2.5, 2.9, 2.10 and 2.11 are convexities with respect to a behaviour.

Example 3.3. Slack $(\alpha, \delta)$-convexity with respect to a network [8].

A particular case of this convexity becomes, after a digitization process, discrete convexity according to the definition of Chassery [2], as proved later in this paper.

Let $(X, d)$ be a totally bounded metric space and for $\varepsilon>0$, let $E^{\prime}$ be a finite $\varepsilon$ network over $X$, and a finite $2 \varepsilon$ network over $E^{\prime}$ is denoted by $E$. For a set $A \subset X$ and a point $x \in X$, the distance between $x$ and $A$ is defined as usual by number $d(x, A)=$ $\inf \{d(x, a) \mid a \in A\}$. In $X$ it is supposed that for every two points $x \in X$ and $y \in X$ the notion of a straight-line segment $\langle x, y\rangle$ is defined (not necessarily the classical one in metric spaces). Let the real numbers $\alpha \geq 0$ and $\delta \geq 0$, and let $E$ be a network chosen as above.

Definition 3.4. Set $A \subset X$ is said to be slackly $(\alpha, \delta)$-convex with respect to $E$ if it is empty or if for each pair of points $x, y \in A$ and for each $t \in\langle x, y\rangle$ if the element $a \in E$ has the property that $d(t, a) \leq \alpha$, then the inequality $d(a, A) \leq \delta$ also holds (Fig. 5).

We take $Y=X, S=\{\{x, y\} \mid x \in X, y \in X\}$ and define two functions $s: S \rightarrow 2^{X}$ and $r: 2^{X} \rightarrow 2^{X}$ by

$$
\begin{aligned}
s(\{x, y\}) & =\{a \in E \mid \exists t \in\langle x, y\rangle, d(a, t) \leq \alpha\}, \quad \text { for every } \quad\{x, y\} \in S, \\
r(C) & =\{t \in X \mid(t, C) \leq \delta\}, \quad \text { for every } \quad C \subseteq X .
\end{aligned}
$$

Theorem 3.2. A nonempty set $A \subseteq X$ is slackly $(\alpha, \delta)$-convex with respect to $E$ if and only if $A$ is $((S, s), r)$ convex. 


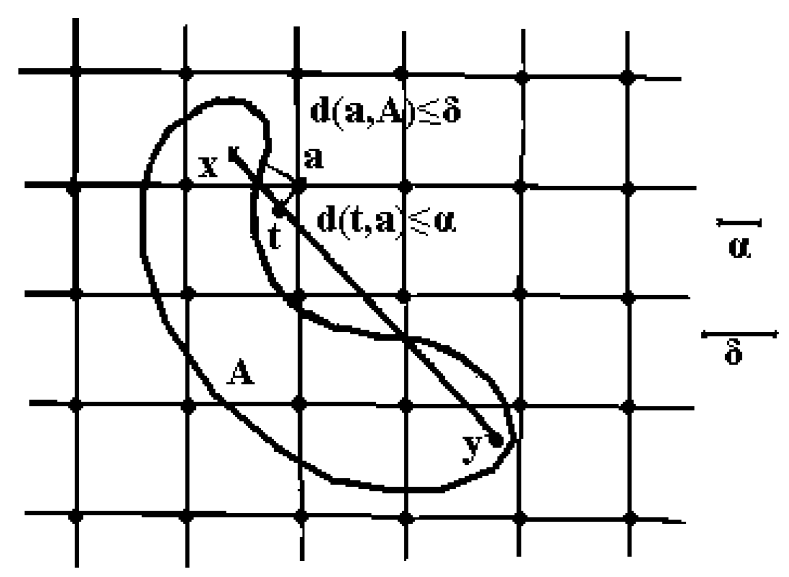

Fig. 5. Slackly $(\alpha, \delta)$-convex set with respect to $Z^{2}$.

Proof. Set $A$ is slackly $(\alpha, \delta)$-convex with respect to $E$ if and only if for each $x, y \in A$ and $t \in\langle x, y\rangle$ the inequality $d(t, a) \leq \alpha$, for $a \in E$, implies $d(a, A) \leq \delta$. This is equivalent to the fact that for each $\{x, y\} \in S$, with $x, y \in A$, one has

$$
\begin{aligned}
s(\{x, y\}) & =\{a \in E \mid t \in\langle x, y\rangle, d(a, t) \leq \alpha\} \\
& \subseteq\{a \in E \mid d(a, A) \leq \delta\} \\
& \subseteq\{t \in X \mid d(t, A) \leq \delta\}=r(A) .
\end{aligned}
$$

Hence $A$ is $((S, s), r)$ convex. Therefore the hypothesis that $A$ is slackly $(\alpha, \delta)$-convex is equivalent to the property of the $((S, s), r)$ convexity of $A$.

It is obvious that the slack $(\alpha, \delta)$-convexity with respect to a set contains discrete cases. For example, set

$$
B=\left\{(0,1),(0,2),(1,0),(1,1),\left(\frac{4}{5}, \frac{11}{5}\right),\left(\frac{9}{5}, 0\right)\right\}
$$

is slackly $\left(\frac{6}{25}, \sqrt{2} / 5\right)$-convex with respect to $\mathbf{Z}^{2}$ if the Euclidean distance is used for measuring.

Example 3.4. Slack convexity with respect to a set and two behaviours [9].

$X$ is assumed to be a nonempty set so that for every two points $x, y$ of $X$ the closed straight-line segment determined by these points, denoted by $\langle x, y\rangle$, is defined. We suppose that $M$ is a nonempty subset of $X$, that $B^{\prime}$ and $B^{\prime \prime}$ are two nonempty sets, that $D^{\prime} \subset B^{\prime}, D^{\prime \prime} \subset B^{\prime \prime}$ are nonempty subsets, and that $\mathcal{T}^{\prime}$ is a set of transformations $T^{\prime}: X \times M \rightarrow B^{\prime}$. The behaviour $C^{\prime}=\left(D^{\prime}, T^{\prime}\right)$ (or a strong one s- $\left(D^{\prime}, \mathcal{T}^{\prime}\right)$, or a weak one $\left.\mathrm{w}-\left(D^{\prime}, \mathcal{T}^{\prime}\right)\right)$ is defined in $X \times M$ by means of $B^{\prime}$. For the set $\mathcal{T}^{\prime \prime}$ containing transformations $T^{\prime \prime}: M \times 2^{X} \rightarrow B^{\prime \prime}$, we define a behaviour $C^{\prime \prime}=\left(D^{\prime \prime}, T^{\prime \prime}\right)\left(\right.$ or s- $\left(D^{\prime}, \mathcal{T}^{\prime \prime}\right)$, or w- $\left.\left(D^{\prime}, \mathcal{T}^{\prime \prime}\right)\right)$ in $M \times 2^{X}$ by means of $B^{\prime \prime}$. 
Definition 3.5. The set $Y \subset X$ is said to be slackly convex with respect to set $M$ and behaviours $C^{\prime}$ and $C^{\prime \prime}$ if it is empty or if for each two points $x, y \in Y$ and for each $t \in\langle x, y\rangle$, if the element $(t, a) \in C^{\prime}(X \times M)$, with $a \in M$, then the element $(a, Y) \in C^{\prime \prime}\left(M \times 2^{X}\right)$.

As above, we take $Y=X, S=\{\{x, y\} \mid x \in X, y \in X\}$ and we define functions $s: S \rightarrow 2^{X}$ and $r: 2^{X} \rightarrow 2^{X}$ by

$$
\begin{aligned}
s(\{x, y\}) & =\left\{a \in M \mid \exists t \in\langle x, y\rangle,(t, a) \in C^{\prime}(X \times M)\right\}, \quad \text { for all } \quad\{x, y\} \in S, \\
r(Z) & =\left\{t \in M \mid(t, Z) \in C^{\prime \prime}\left(M \times 2^{X}\right)\right\}, \quad \text { for all } \quad Z \subseteq X .
\end{aligned}
$$

Then it is easy to prove that a nonempty set $A \subseteq X$ is convex with respect to set $M$ and behaviours $C$ and $C^{\prime \prime}$ if and only if $A$ is $((S, s), r)$ convex. Also, it is evident that Example 3.3 presents a convexity that is a particular case of slack convexity with respect to a set and two behaviours. Other particular cases are the convexities defined in Examples 2.5, 2.9, 2.10 and 2.11.

Example 3.5. Induced $k$-strong convexity with respect to $p, f$ and $M$ [1].

Let $X, T$ be two arbitrary sets, let $M$ be a nonempty subset of $T$ and let us consider functions $f: X \rightarrow T$ and $p: 2^{T} \rightarrow 2^{T}$. Let $k \geq 2$ be a natural number.

Definition 3.6. A subset $A \subseteq X$ is called induced $k$-strongly convex with respect to $p, f$ and $M$ if $p\left(\left\{f\left(a_{1}\right), \ldots, f\left(a_{k}\right)\right\}\right) \cap M \subseteq f(A)$, for any $a_{1}, \ldots, a_{k} \in A$.

Now, we take $Y=X, S=\left\{\left\{a_{1}, \ldots, a_{k}\right\} \mid a_{j} \in X, j \in\{1,2, \ldots, k\}\right\}$ and define functions $s: S \rightarrow 2^{X}$ and $r: 2^{X} \rightarrow 2^{X}$ by $s(B)=p\left(\left\{f\left(a_{1}\right), \ldots, f\left(a_{k}\right)\right\}\right) \cap M$, $r(C)=f(C)$, for $B=\left\{a_{1}, \ldots, a_{k}\right\} \in S$ and $C \in 2^{X}$. It is easy to prove that a set $A \subseteq X$ is induced $k$-strongly convex with respect to $p, f$ and $M$ if and only if it is $((S, s), r)$ convex.

There are discrete sets, which are induced $k$-strongly convex with respect to $p, f$ and $M$. For example, let $X=Y=\mathbf{R}^{2}, M=\mathbf{Z}^{2}, f=1_{\mathbf{R}^{\mathbf{n}}}$, and let $p: 2^{Y} \rightarrow 2^{Y}$, $p(B)=\operatorname{conv}(B)$, for each $B \in 2^{Y}$. If we take $A=\{(1,3),(0,2),(2,1)\}$, then $A$ is induced 2-strongly convex with respect to $p, f$ and $M$.

\section{Special Partial $((S, s), r)$ Convexity Concepts}

\subsection{Special Partial $((S, s), r)$ Discrete Convexity Concepts}

The convexity properties from this class are obtained by a special segmental method, which looks into the given set through a network (as in the case of a digitization process).

Supposing that the $\mathcal{S}$ family is a singleton, $\mathcal{S}=\{s\}$ with $s: S \rightarrow 2^{Y}$, and the $\mathcal{R}$ family is also a singleton, $\mathcal{R}=\{r\}$, with $r: 2^{X} \rightarrow 2^{Y}$, we define $((S, s), r)$ convexity as follows: 
Definition 4.1. A set $A \subseteq X$ is said to special partially $((S, s), r)$ convex if $A \in S$ and $s(A) \subseteq r(A)$.

Therefore, special partial convexity supposes that condition $s(C) \subseteq r(A)$ holds for $C=A$.

Example 4.1. Convexity of cellular blobs [36].

Usually, a figure is the union of a simple closed curve and its interior. A blob is any bounded figure. A figure is convex provided that it is classically convex. A cellular mosaic is a set of bounded convex figures $\left\{c_{i}\right\}$, called cells, such that $c_{i} \cap c_{j}$ equals either $\emptyset$ or a part of the boundary of $c_{j}$, for all $i \neq j$, and such that the union of all the cells covers the entire plane. A cellular map is a nonempty subset of cells of a cellular mosaic.

Suppose that $J$ is a cellular map. The boundary of $J$, denoted by $b(J)$, is the boundary of the union of the cells of $J$. The cellular boundary of $J$, denoted by $B(J)$, is the union of cells, each of which belongs to $J$ and contains a point of $b(J)$. Let $\gamma(J)$ denote a nonrepeating closed chain that contains precisely the cells of $B(J)$. Consider the map $g(J)$ formed by the union of the boundaries of the cells of $\gamma(J)$, excluding the boundaries between successive cells of $\gamma(J)$. The core boundary of $J$, denoted by $b C(J)$, is the set of all points in $g(J)$ that are not connected to $b(J)$. The union of $b C(J)$ and its interior is the core of $J$, denoted by $C(J)$. $J$ is cored if $C(J) \neq \emptyset$.

A cellular blob is a bounded, cored cellular map.

A cellular map $J$ is the cellular image (or briefly the image) of a figure $p$ if and only if either

(a) the union of the members of $J$ contains $p$ and

(b) every member of $J$ containing an exterior point of $p$ also contains a boundary point of $p$, or $p$ is the limit of a sequence of figures each of which satisfies (a) and (b).

Definition 4.2. A cellular blob is convex if there is at least one convex figure $r$ of which the given cellular blob is an image (Fig. 6).

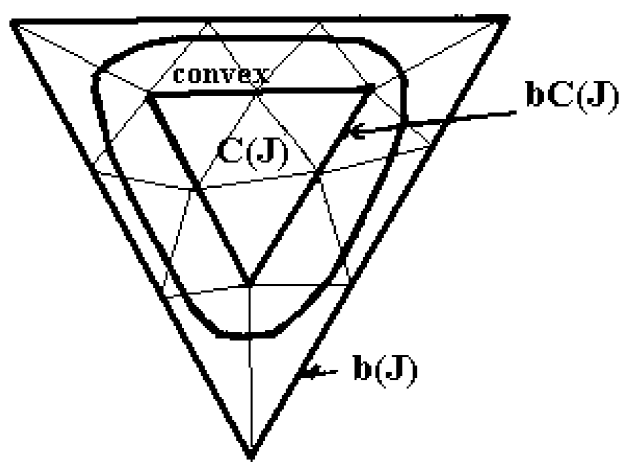

Fig. 6. Convex cellular blob. 
$F$ denotes the set of all plane figures. $C B$ denotes the set of all cellular blobs. A digitization process is a function $I: F \rightarrow C B$, such that $I(a)=J$, for $a \in F$ and $J \in C B$ is the cellular image of figure $a$. We denote $I^{-1}(J)=\{a \in F \mid I(a)=J\}$ and $C F=\{a \in F \mid a$ is classically convex $\}$.

Now, we take $X=Y=2^{\mathbf{R}^{2}}, S=F, s: S \rightarrow 2^{Y}, s(a)=I(a)$, if $a \in C F \cap I^{-1}(C B)$, and $s(a)=X$, for $a \in S \backslash C F \cap I^{-1}(C B)$, and $r=1_{2^{X}}$. Then it is obvious that a cellular blob $J$ is convex if there is an element $a \in C F \cap I^{-1}(C B)$ such that $s\left(I^{-1}(J)\right)=$ $I(a)=J=r(J)$.

Theorem 4.1. A cellular blob $J$ is convex if and only if $J$ is special partial $((S, s), r)$ convex.

Example 4.2. Digital convexity in $\mathbf{Z}^{2}$ [18].

Kim [18] defined a similar notion of discrete convexity in $\mathbf{Z}^{2}$, using the following model. Let $A$ be a subset of a plane such that $\operatorname{cl}(\operatorname{int}(A))=A(A$ is the closure of its interior). This type of set is called regular. Each lattice point $p \in \mathbf{Z}^{2}$ is regarded as the centre of an open unit square (cell), $p^{*}$. The set $I(A)=\left\{p \mid A \cap p^{*} \neq \emptyset\right\}$ is called the digital image of $A$.

Definition 4.3. A set $A \subseteq \mathbf{Z}^{2}$ is digitally convex if it is the digital image of a convex regular set $R \subseteq \mathbf{Z}^{2}$.

It is evident that digital convexity is another special partial $((S, s), r)$ convexity. It is a convexity of cellular blobs, with particular cells, i.e. unit squares.

Example 4.3. Discrete convexity in $\mathbf{Z}^{2}$ [20].

Another discrete convexity notion is the cellular convexity introduced by Kim and Sklansky [20]. Even if it is equivalent to the convexity of cellular blobs, it is a distinct convexity notion referring to sets of rectangular cells in the plane, called cellular complexes, instead of sets of general cells. In order to define the convexity of discrete images in $\mathbf{Z}^{2}$, a network expressed by reducing the cells to some typical figures (squares) covers the plane. So, a mosaic, isomorphic to $\mathbf{Z}^{2}$, covers the plane.

Definition 4.4. A connected component $A$ is said to have cellular convexity if and only if there is at least one convex figure in $\mathbf{R}^{2}$ that might become $A$ after a digitization process.

It is obvious that this convexity belongs to the same class of properties as the convexity of cellular blobs.

Example 4.4. Fuzzy digital convexity [17].

Let $E$ be the Euclidean plane (it is possible to be a set in which the straight-line segment is defined and each straight-line is a totally ordered set). A subset $M$ of $E$ is 
said to be regular if it coincides with the closure of its interior, $M=\operatorname{cl}(\operatorname{int}(M))$. As in Example 3.4, each lattice point $p \in \mathbf{Z}^{2}$ is regarded as the centre of an open unit square (cell), $p^{*}$. Set $I(A)=\left\{p \mid A \cap p^{*} \neq \emptyset\right\}$ is called the digital image of $A$. A fuzzy set $A$ in $E$ is represented by a characteristic function $\chi_{A}: E \rightarrow[0,1]$. Given a fuzzy subset $A$ of the plane, we define a fuzzy subset $A^{\prime}$ of $\mathbf{Z}^{2}$ represented by the characteristic function $\chi_{A}^{\prime}(p)=\sup \left\{\chi_{A}(x) \mid x \in p^{*}\right\}$. If $A$ is regular, then $A^{\prime}$ is its digital image (as in Example 3.3). A fuzzy set $A$ is fuzzily regular if all its level sets $A_{t}, 0 \leq t<1$, are regular.

Definition 4.5. A fuzzy subset $A^{\prime}$ of $\mathbf{Z}^{2}$ is fuzzily digitally convex if it is the digital image of a fuzzily regular, fuzzily convex fuzzy set $A$.

Obviously, fuzzy digital convexity is a special partial $((S, s), r)$ convexity.

\subsection{Special Partial $((S, s), r)$ Convexities Having Discrete Particular Cases}

Example 4.5. Induced strong convexity with respect to $p, f$ and $M[1]$.

Let $X, T$ be two arbitrary sets, let $M$ be a nonempty subset of $T$ and let us consider functions $f: X \rightarrow T$ and $p: 2^{T} \rightarrow 2^{T}$.

Definition 4.6. A subset $A \subseteq X$ is said to be induced strongly convex with respect to $p, f$ and $M$ if

$$
p(f(A)) \cap M \subseteq f(A) .
$$

We take $Y=X, S=2^{X}$ and define $s: S \rightarrow 2^{X}$ and $r: 2^{X} \rightarrow 2^{X}$ by $s(B)=$ $p(f(B)) \cap M$ and $r(A)=f(A)$, for $B \in S$ and $A \in 2^{X}$. Now it is easy to prove that a set $A \subseteq X$ is induced strongly convex with respect to $p, f$ and $M$ if and only if it is special partial $((S, s), r)$ convex.

There are discrete sets having a convexity property of this type. For example, let $X=$ $Y=\{-1,0,1,2\}, f=1_{X}, M=X$ and let $p: 2^{Y} \rightarrow 2^{Y}$, given by $p(\emptyset)=\emptyset, p(\{-1\})=$ $\{1\}, p(\{0\})=\{0\}, p(\{1\})=\{1\}, p(\{2\})=\{2\}, p(\{-1,0\})=\{1\}, p(\{-1,1\})=\{1\}$, $p(\{-1,2\})=\{2\}, p(\{1,2\})=\{1,2\}, p(\{-1,0,1\})=\{0,1\}, p(\{-1,0,2\})=\{0,2\}$, $p(\{-1,1,2\})=\{1,2\}, p(\{0,1,2\})=\{0,1,2\}, p(\{-1,0,1,2\})=\{0,1,2\}$. Because

$$
p(f(\{-1,0,1\})) \cap M=\{0,1\} \subseteq\{-1,0,1\}=f(\{-1,0,1\}),
$$

it follows that set $A=\{-1,0,1\}$ is induced strongly convex with respect to $p, f$ and $M$.

\section{5. $(e, a)-((S, \mathcal{S}), r)$ Convexity Concepts}

\section{1. $(e, a)-((S, \mathcal{S}), r)$ Discrete Convexity Concepts}

This class also contains convexity properties obtained by looking to a set through a network in order to obtain an approximation of classical convexity. We include here the 
three-point convexity of Valentine, which is at the origin of an important number of applications leading to more concepts of discrete convexity.

Supposing that the $\mathcal{R}$ family is a singleton, $\mathcal{R}=\{r\}$, with $r: 2^{X} \rightarrow 2^{Y}$, we define $(e, a)-((S, \mathcal{S}), r)$ convexity as follows:

Definition 5.1. A set $A \subseteq X$ is said to be $(e, a)-((S, \mathcal{S}), r)$ convex if for every subset $C \in S, C \subseteq A$, there is a function $s \in \mathcal{S}$ which satisfies the inclusion $s(C) \subseteq r(A)$.

The letter " $e$ " from the pair $(e, a)$ means that the $\mathcal{S}$ family appears accompanied by the existential quantifier $(\exists s \in \mathcal{S})$. The letter " $a$ " from the pair $(e, a)$ means that the $\mathcal{R}$ family appears accompanied by the universal quantifier $(\forall r \in \mathcal{R})$.

The following examples present all the $(e, a)-((S, \mathcal{S}), r)$ discrete convexities, i.e. the $(e, a)-((S, \mathbf{S}), r)$ convexity properties of sets in the case $X=\mathbf{Z}^{2}$ or $X$ is a discrete network over a totally bounded metric space.

Example 5.1. Three-point convexity [37].

Three-point convexity is neither a discrete convexity nor contains nontrivial (sets containing more that two distinct points) discrete particular cases. However, it is the origin of an important point of view on digital convexity, with more applications, and this is the reason for its presence in our paper.

$E_{n}$ is supposed to be the $n$-dimensional Euclidean space and $A \subseteq E_{n}$. Set $A$ is said to have the three-point property $P_{3}$ if for each triple of points $x, y, z$ in $A$ at least one of the closed segments $\langle x, y\rangle,\langle x, z\rangle,\langle y, z\rangle$ is in $A$.

Definition 5.2. $\quad$ A set $A$ is three-point convex if $A$ possesses the property $P_{3}$.

We take $X=E_{n}, Y=E_{n} \bigcup\left\{0_{n+1}\right\}$, where $0_{n+1}=(0, \ldots, 0) \in E_{n+1}$, and $S=$ $\{\{x, y, z\} \mid x \in X, y \in X, z \in X\}$. Function $r$ is the identity on $2^{Y}$ and the set of functions $s$ is defined by $\mathcal{S}=\left\{s_{x, y} \mid s_{x, y}: S \rightarrow 2^{Y}, x \in X, y \in Y\right\}$, where

$$
s_{x, y}(\{a, b, c\})=\left\{\begin{array}{cll}
\langle x, y\rangle, & \text { if }\{x, y\} \subseteq\{a, b, c\}, & \text { for each } \quad x, y \in X . \\
\left\{0_{n+1}\right\}, & \text { otherwise, }
\end{array}\right.
$$

Theorem 5.1. A set $A \subseteq E_{n}$ is three-point convex if and only if $A$ is $(e, a)-((S, \mathcal{S}), r)$ convex.

Proof. The proof is evident if we notice that, for each triple $x, y, z \in A$, the existence of two of them, say $a$ and $b$, such that $\langle a, b\rangle \subset A$ is equivalent to the existence of a function $s=s_{a, b}$ satisfying

$$
s_{a, b}(\{x, y, z\})=\langle a, b\rangle \subseteq A=r(A) .
$$

This means that $A$ is $(e, a)-((S, \mathcal{S}), r)$ convex.

Example 5.2. Collinear three-point convexity $\left(\mathrm{CP}_{3}\right)$-convexity) [21]. 
Collinear three-point convexity is obtained by introducing the condition of collinearity in the definition of property $P_{3}$ defined by Valentine [37]. Collinearity was necessary in connection with the problem of the recognition of convexity by directional tests. The notion of $\mathrm{CP}_{3}$-convexity was defined as a plane property, even though it might be discussed in a more general framework. We keep the original framework. Let $X=\mathbf{R}^{2}$ and $A \subset \mathbf{R}^{2}$. We say that set $A$ has property $C P_{3}$ (collinear $P_{3}$ ) if $P_{3}$ holds for all collinear triples of points of $A$.

Definition 5.3. A set $A$ is $C P_{3}$-convex if it has property $C P_{3}$.

We take $X=E_{n}, Y=E_{n} \bigcup\left\{0_{n+1}\right\}$, where $0_{n+1} \in E_{n+1}$, and $S=\{\{x, y, z\} \mid x \in$ $X, y \in X, z \in X, x, y, z$ collinear\}. Function $r$ is the identity on $2^{Y}$ and the set of functions $s$ is defined by $\mathcal{S}=\left\{s_{x, y} \mid s_{x, y}: S \rightarrow 2^{Y}, x \in E_{n}, y \in E_{n}\right\}$ where, for each $x, y \in E_{n}$,

$$
s_{x, y}(\{a, b, c\})=\left\{\begin{aligned}
\langle x, y\rangle, & \text { if }\{x, y\} \subseteq\{a, b, c\}, \\
\left\{0_{n+1}\right\}, & \text { otherwise. }
\end{aligned}\right.
$$

Theorem 5.2. A set $A \subseteq E_{n}$ is $C P_{3}$-convex if and only if $A$ is $(e, a)-((S, \mathcal{S}), r)$ convex.

The proof is similar to the previous one.

Example 5.3. Discrete convexity [2], [3].

In the plane $\mathbf{R}^{2}$ we consider the chessboard distance $c(x, y)=\max \left(\left|x_{1}-y_{1}\right|,\left|x_{2}-y_{2}\right|\right)$ for $x=\left(x_{1}, x_{2}\right) \in \mathbf{R}^{2}, y=\left(y_{1}, y_{2}\right) \in \mathbf{R}^{2}$. For the real positive number $h$, we denote by

$$
\mathbf{Z}^{2}(h)=\{(i h, j h) \mid i \in \mathbf{Z}, j \in \mathbf{Z}\}
$$

the set of the lattice points of the grid of step $h$ over the plane.

Definition 5.4. A set $A \subset \mathbf{Z}^{2}(h)$ is $\varepsilon$ convex, for $\varepsilon \in[h / 2, h[$, if for every $x, y \in A$ and $t \in] 0,1\left[\right.$, there is a point $a \in \mathbf{Z}^{2}(h)$ such that $c(t x+(1-t) y, a) \leq \varepsilon$.

Definition 5.5. A set $A \subset \mathbf{Z}^{2}(h)$ is discrete convex if there is a real number $\varepsilon \in[h / 2, h[$ such that $A$ is $\varepsilon$ convex.

In what follows, for each $z \in \mathbf{R}^{2}$ and for each $\alpha \in[h / 2, h$, we put

$$
M_{z, \alpha}=\{m \mid m \in E, d(z, m) \leq \alpha\} .
$$

We take $X=Y=\mathbf{Z}^{2}(h), S=\left\{\{x, y\} \mid x \in \mathbf{Z}^{2}(h), y \in \mathbf{Z}^{2}(h)\right\}$ and we say that function $s: S \rightarrow 2^{X}$ has the property $(P)$ if

$$
s(\{x, y\})= \begin{cases}X, & \text { if }\langle x, y\rangle=\emptyset \\ \bigcup_{z \in\langle x, y\rangle}\left\{u_{z}\right\} & \text { or there is } z \in\langle x, y\rangle \text { such that } M_{z, \alpha}=\emptyset,\end{cases}
$$


where $u_{z} \in M_{z, \alpha}$ for all $z \in\langle x, y\rangle$. We denote $\mathcal{S}=\left\{s \mid s: S \rightarrow 2^{X}\right.$, s has property $\left.(P)\right\}$. We define function $r: 2^{X} \rightarrow 2^{X}$ by $r(B)=B$, for all $B \in 2^{X}$.

Theorem 5.3. Set $A \subset \mathbf{Z}^{2}(h)$ is discrete convex if and only if it is $(e, a)-((S, \mathcal{S}), r)$ convex.

The proof is similar to the proof of Theorem 5.1.

Further properties of this discrete convexity, together with applications in the study of cytology tests, are presented in [4].

Remark 3.1 shows that each discrete convex set in $\mathbf{Z}^{2}$ is integer convex, but the converse is not true, as the example of set $A=\{(0,0),(1,3)\}$ shows.

Example 5.4. Discrete convexity [19].

Kim and Rosenfeld [20] published a constructive geometrical approach for sets of knots of a network of type $\mathbf{Z}^{2}(h)$ using the chessboard distance $c(x, y)=\max \left\{\mid x_{1}-\right.$ $\left.y_{1}|,| x_{2}-y_{2} \mid\right\}, x=\left(x_{1}, x_{2}\right) \in \mathbf{Z}^{2}(h), y=\left(y_{1}, y_{2}\right) \in \mathbf{Z}^{2}(h)$. Let $A$ be an 8 -connected component. $A^{\prime}$ denotes the set of all the squares of side $h>0$ centred in the points of $S$. The subset of $\mathbf{R}^{2}$ containing all the points of $A^{\prime}$ is denoted by $p(A)$. Let $b p(A)$ be its frontier. For every two points of $A, P_{1}$ and $P_{2}$, the set of those polygons having the frontiers made both by parts of $\left\langle P_{1}, P_{2}\right\rangle$ and $b p(A)$ and the interiors in $p(A)$ is denoted by $P\left(A ; P_{1}, P_{2}\right)$. The convexity notion defined by the two authors is:

Definition 5.6. A connected component $A$ is said to be convex if for every pair of points of $A, P_{1}$ and $P_{2}$, the subset $P\left(A ; P_{1}, P_{2}\right)$ does not contain points from the complement of $A$.

Therefore, we must have $P\left(A ; P_{1}, P_{2}\right) \cap \mathbf{Z}^{2}(h) \subseteq A$.

We take $X=\mathbf{Z}^{2}(h), Y=\mathbf{R}^{2}$ and $S=\left\{\{x, y\} \mid x, y \in \mathbf{Z}^{2}(h)\right\}$. The family $\mathcal{S}=\left\{s_{A} \mid\right.$ $\left.s_{A}: S \rightarrow 2^{Y}\right\}$, with

$$
s_{A}(\{x, y\})= \begin{cases}P(A ; x, y) \cap \mathbf{Z}^{2}(h), & \text { if } \quad\{x, y\} \subset A, \\ X, & \text { if } \quad\{x, y\} \nsubseteq A,\end{cases}
$$

and function $r: 2^{X} \rightarrow 2^{Y}$ is defined by $r(A)=A$ for each $A \subseteq \mathbf{Z}^{2}(h)$.

Now, it is obvious that a set $A \subseteq \mathbf{Z}^{2}(h)$ is convex if and only if $A$ is $(e, a)-((S, \mathcal{S}), r)$ convex.

\section{2. (e, a)- $((S, S), r)$ Convexities Having Discrete Particular Cases}

Example 5.5. Strong $(\alpha, \delta)$-convexity with respect to a network [8].

Let $(X, d)$ be a totally bounded metric space and for $\varepsilon>0$, let $E^{\prime}$ be a finite $\varepsilon$ network over $X$. A finite $2 \varepsilon$ network over $E^{\prime}$ is denoted by $E$. For a set $A \subset X$ and a point $x \in X$, the distance between $x$ and $A$ is defined as usual by the number $d(x, A)=\inf \{(x, a) \mid$ 


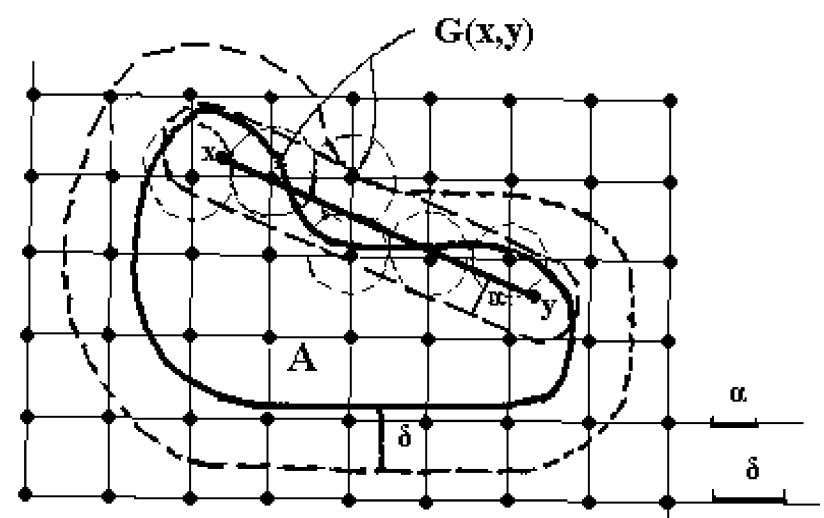

Fig. 7. Set $A$ is strongly $(\alpha, \delta)$-convex with respect to $Z^{2}$.

$a \in A\}$. In $X$ it is supposed that, for every two points $x \in X$ and $y \in X$, a notion of a straight-line segment $\langle x, y\rangle$ is defined (it might be distinct from the metric straight-line segment).

We fix the real numbers $\alpha>0$ and $\delta>0$, and let $E$ be a network chosen as above.

Definition 5.7. Set $A \subset X$ is said to be strongly ( $\alpha, \delta)$-convex with respect to $E$ if it is empty or for every pair of points $x, y \in A$ and for every $t \in\langle x, y\rangle$ there is an element $a \in E$ such that $d(t, a) \leq \alpha$ and $d(a, A) \leq \delta$.

In other words, a set $A \subseteq X$ is strongly $(\alpha, \delta)$-convex with respect to $E$ if for each pair of points $x \in A, y \in A$, there is a subset $G(x, y)$ of points of $E$ situated in an $\alpha$-neighbourhood of segment $\langle x, y\rangle$ simultaneously having the properties:

1. The union of all the closed balls centred in points of $G(x, y)$ and of radius $\alpha$ cover $\langle x, y\rangle$.

2. Each point of $G(x, y)$ lies in the $\delta$-neighbourhood of set $A$ (Fig. 7).

This property contains discrete particular cases and is distinct from the slack $(\alpha, \delta)$ convexity with respect to $E$ from Definition 3.4. Indeed, set $A=\{(0,1),(1,0),(2,0)\}$ is strongly $\left(\frac{2}{3}, \frac{1}{2}\right)$-convex with respect to $\mathbf{Z}^{2}$, without being slackly $\left(\frac{2}{3}, \frac{1}{2}\right)$-convex with respect to $\mathbf{Z}^{2}$. If points $x=(0,1), y=(2,0)$ and $t=\left(1, \frac{1}{2}\right) \in\langle x, y\rangle$ are taken into account, then point $a=(1,1)$ has the property that $c(t, a)=\frac{1}{2}$, but $c(a, A)=1>\frac{1}{2}$, where $c$ means the Chebyshev distance in the plane. On another hand, Example 5.3.1 on p. 118 of [16] describes a slackly $(\alpha, \delta)$-convex set with respect to $\mathbf{Z}^{2}$, which is not strongly $(\alpha, \delta)$-convex set with respect to $\mathbf{Z}^{2}$.

In what follows, for each $z \in X$ we put $M_{z}=\{m \mid m \in E, d(z, m) \leq \alpha\}$. We take $S=\{\{x, y\} \mid x \in, \mathrm{y} \in\}$ and we say that function $s: S \rightarrow 2^{X}$ has the property $(P)$ if

$$
s(\{x, y\})= \begin{cases}X, & \text { if }\langle x, y\rangle=\emptyset \\
\bigcup_{z \in\langle x, y\rangle}\left\{u_{z}\right\} & \begin{array}{l}
\text { or there is } z \in\langle x, y\rangle \quad \text { such that } \quad M_{z, \alpha}=\emptyset, \\
\text { in other cases, }
\end{array}\end{cases}
$$


where $u_{z} \in M_{z}$, for all $z \in\langle x, y\rangle$. We put $\mathcal{S}=\left\{s \mid s: S \rightarrow 2^{X}, s\right.$ has property $(P)\}$. We define function $r: 2^{X} \rightarrow 2^{X}$ by $r(B)=\{m \mid m \in E, d(m, B) \leq \delta\}$, for all $B \in 2^{X}$.

Theorem 5.4. Set $A \subset X$ is strongly $(\alpha, \delta)$-convex with respect to $E$ if and only if it is $(e, a)-((S, \mathcal{S}), r)$ convex.

Proof. We suppose that set $A \subset X$ is strongly $(\alpha, \delta)$-convex with respect to $E$. Then for each pair of points $x, y \in A$ and for each $t \in\langle x, y\rangle$ there is an element $a \in E$ such that the following conditions hold simultaneously: $d(t, a) \leq \alpha$ and $d(a, A) \leq \delta$. It means that for each pair of points $x, y \in A$ and for each $t \in\langle x, y\rangle$ there is an element $a \in M_{t}$ and then $s(\{x, y\})=\bigcup_{t \in\langle x, y\rangle}\left\{u_{t}\right\}$. At the same time, each element $a \in \bigcup_{t \in\langle x, y\rangle}\left\{u_{t}\right\}$ also belongs to $r(A)$, meaning that $s(\{x, y\}) \subseteq r(A)$. Therefore, $A$ is $(e, a)-((S, \mathcal{S}), r)$ convex.

Conversely, if $A$ is $(e, a)-((S, \mathcal{S}), r)$ convex, then for each $\{x, y\} \in S$ and $\{x, y\} \subseteq A$, there is a function $s \in \mathcal{S}$ such that $s(\{x, y\}) \subseteq r(A)$. It is obvious that $s(\{x, y)\} \neq X$ because $r(A) \subseteq E \subset X$. Therefore $s(\{x, y\})=\bigcup_{t \in\langle x, y\rangle}\left\{u_{t}\right\}$ and then for each $t \in\langle x, y\rangle$ there is an element $a \in M_{t}$, meaning that $d(t, a) \leq \alpha$. The inclusion $s(\{x, y\}) \subseteq r(A)$ implies that $d(a, A) \leq \delta$ also occurs. Therefore, $A$ is strongly $(\alpha, \delta)$-convex with respect to $E$.

A particular case of this convexity becomes, after a digitization process, discrete convexity in the sense of the definition of Chassery [2], as is shown in the following result. Recall that a function $f: \mathbf{R}^{2} \rightarrow \mathbf{Z}^{2}(h)$ with the property that, for every $x \in \mathbf{Z}^{2}(h)$, $f(x)=x$ occurs is said to be a method of digitization of $\mathbf{R}^{2}$ into $\mathbf{Z}^{2}(h)$.

Theorem 5.5. If a set $A \subset \mathbf{R}^{2}$ is both strongly and slackly $(\varepsilon, 0)$-convex with respect to $\mathbf{Z}^{2}(h)$, for $\varepsilon \in\left[h / 2, h\right.$, then there is a method of digitization $f: \mathbf{R}^{2} \rightarrow \mathbf{Z}^{2}(h)$ such that $f(A)$ is discrete convex.

Proof. Consider the method of digitization defined by $f(a)=(x, y)$ if $a \in] x-$ $h / 2, x+h / 2] \times] y-h / 2, y+h / 2]$, for every $(x, y) \in \mathbf{Z}^{2}(h)$ and $a \in \mathbf{R}^{2}$. Suppose that $A$ is both strongly and slackly $(\varepsilon, 0)$-convex with respect to $\mathbf{Z}^{2}(h)$. Then for every $x, y \in A$ and $t \in] 0,1$ [ there is an $a \in \mathbf{Z}^{2}(h)$ such that $c(t x+(1-t) y, a) \leq \varepsilon$ involves that $a \in A$. To prove that $f(A)$ is discrete convex we suppose that $x$ and $y$ are two points of $f(A)$. Two situations are possible:

Case 1. $x \in A \cap f(A)$ and $y \in A \cap f(A)$.

Case 2. $x$ or $y$ belongs to $f(A) \backslash A$.

Case 1. If $x, y \in A \cap f(A)$, then from the strongly $(\varepsilon, 0)$-convexity of $A$ with respect to $\mathbf{Z}^{2}(h)$ it follows that for every $\left.t \in\right] 0,1\left[\right.$ there is an $a \in \mathbf{Z}^{2}(h)$ such that $c(t x+(1-$ t) $y, a) \leq \varepsilon$ involves that $a \in A$. However, $a \in A$ and $a \in \mathbf{Z}^{2}(h)$ means that $a \in f(A)$ and, therefore, the definition of discrete convexity is fulfilled. 
Case 2. Now $x \in f(A) \backslash A$ and $y \in A$ are supposed. Then two situations are possible in the neighbourhood of $x$ :

(a) There is $z \in A$ such that $c(x, z)<h / 2$.

(b) The closest point $z \in A$ satisfies $c(x, z)=h / 2$.

(a) If $z \in A$ is a point such that $c(x, z)<h / 2$, then there is a point $u \in\langle x, y\rangle$ satisfying $\langle z, u\rangle \subseteq \operatorname{conv}\left(B_{\infty}(z, h / 2)\right)$ and $\langle u, y\rangle \nsubseteq \operatorname{conv}\left(B_{\infty}(x, h / 2)\right)$. If $\left.t \in\right] 0,1[$ is chosen such that $t z+(1-t) y \in\langle z, u\rangle$, then $c(t z+(1-t) y, x)<h / 2 \leq \varepsilon$. Therefore, from the slackly $(\varepsilon, 0)$-convexity of $A$ with respect to $\mathbf{Z}^{2}(h)$ it follows that $x \in A$ and the hypothesis of case 2 cannot occur.

(b) The slack $(\varepsilon, 0)$-convexity of $A$ with respect to $\mathbf{Z}^{2}(h)$ has as a consequence that $x \in A$, by taking $t=0$ in the reasoning above. Therefore the hypothesis of case 2 cannot hold.

The cases $x \in f(A), y \in f(A) \backslash A$ and $x \in f(A) \backslash A, y \in f(A) \backslash A$ are similar.

This theorem proves that all the convexity properties with respect to a set and two behaviours have discrete particular cases: for example, the discrete convexity of Chassery is one of them.

Theorem 5.5 remains valid if another distance is used in the space instead of the chessboard metric.

Example 5.6. Strong convexity with respect to a set and two behaviours [9].

$X$ is assumed to be a nonempty set so that for every two points $x, y$ of $X$ the closed straight-line segment determined by these points, denoted by $\langle x, y\rangle$, is defined. We suppose that $M$ is a nonempty subset of $X$, that $B^{\prime}$ and $B^{\prime \prime}$ are two nonempty sets, that $D^{\prime} \subset B^{\prime}, D^{\prime \prime} \subset B^{\prime \prime}$ are nonempty subsets, and that $\mathcal{T}^{\prime}$ is a set of transformations, $T^{\prime}: X \times M \rightarrow B^{\prime}$. Behaviour $C^{\prime}=\left(D^{\prime}, T^{\prime}\right)$ (or a strong one s- $\left(D^{\prime}, \mathcal{T}^{\prime}\right)$, or a weak one w$\left.\left(D^{\prime}, \mathcal{T}^{\prime}\right)\right)$ is defined in $X \times M$ by means of $B^{\prime}$. For set $\mathcal{T}^{\prime \prime}$ of transformations, $T^{\prime \prime}: M \times$ $2^{X} \rightarrow B^{\prime \prime}$, we define a behaviour $C^{\prime \prime}=\left(D^{\prime \prime}, T^{\prime \prime}\right)\left(\right.$ or s- $\left(D^{\prime}, \mathcal{T}^{\prime \prime}\right)$, or $\left.\mathrm{w}-\left(D^{\prime}, \mathcal{T}^{\prime \prime}\right)\right)$ in $M \times 2^{X}$ by means of $B^{\prime \prime}$.

Definition 5.8. A set $A \subset X$ is said to be strongly convex with respect to set $M$ and behaviours $C^{\prime}$ and $C^{\prime \prime}$ if it is empty or if for each pair of points $x, y \in A$ and for each $t \in\langle x, y\rangle$ there is an element $a \in M$ such that the following conditions occur simultaneously: $(t, a) \in C^{\prime}(X \times M)$ and $(a, A) \in C^{\prime \prime}\left(M \times 2^{X}\right)$.

In what follows, for each $z \in X$ we put $M_{z}=\left\{m \mid m \in M,(z, m) \in C^{\prime}(X \times M)\right\}$. We take

$$
S=\{\{x, y\} \mid x \in X, y \in X\},
$$

and we say that function $s: S \rightarrow 2^{X}$ has the property $(P)$ if

$$
s(\{x, y\})= \begin{cases}X, & \text { if }\langle x, y\rangle=\emptyset \\ \bigcup_{z \in\langle x, y\rangle}\left\{u_{z}\right\} & \text { or there is } z \in\langle x, y\rangle \text { such that } M_{z}=\emptyset,\end{cases}
$$


where $u_{z} \in M_{z}$, for all $z \in\langle x, y\rangle$. We put $\mathcal{S}=\left\{s \mid s: S \rightarrow 2^{X}\right.$, s has property $\left.(P)\right\}$. We define function $r: 2^{X} \rightarrow 2^{X}$ by $r(B)=\left\{m \mid m \in M,(m, B) \in C^{\prime \prime}\left(M \times 2^{X}\right)\right\}$, for all $B \in 2^{X}$.

Then it is easy to prove as above that set $A \subset X$ is strongly convex with respect to set $M$ and behaviours $C^{\prime}$ and $C^{\prime \prime}$ if and only if it is $(e, a)-((S, \mathcal{S}), r)$ convex. Also, it is easy to prove that the previous example presents a particular case of strong convexity with respect to a set $M$ and two behaviours, following the method described in Example 3.2. Applications of these convexity properties are suggested in [10] and [13]-[16].

Each convexity from Section 2, except those from Examples 2.5, 2.9, 2.10 and 2.11 are properties of this type. Also, Examples 3.1 and 3.2 could be modelled as in Definition 5.8.

\section{Special Partial $(a, e)-((S, \mathcal{S}), \mathcal{R})$ Convexity Concepts with Discrete Particular Cases}

This subclass contains the directional segmental convexities obtained by digitization of the fuzzy directional convexity and concavity. Therefore, in this case only some types of straight-line segments are taken into account: those that are either horizontal or vertical.

The following examples present all the special partial $(a, e)-((S, \mathcal{S}), \mathcal{R})$ discrete convexities, i.e. the special partial $(a, e)-((S, \mathcal{S}), \mathcal{R})$ convexity properties of sets in the case $X=\mathbf{Z}^{2}$ or $X$ is a discrete network over a totally bounded metric space.

Definition 6.1. A set $A \subseteq X$ is said to be special partially $(a, e)-((S, \mathcal{S}), \mathcal{R})$ convex if $A \in S$ and for each function $s \in \mathcal{S}$ there is a transformation $r \in \mathcal{R}$ which satisfies the inclusion $s(A) \subseteq r(A)$.

This class of discrete convexity contains especially fuzzy discrete convexities and fuzzy discrete concavities.

Example 6.1. Fuzzy ortho-convexity [5].

Let $E$ be the Euclidean plane.

Definition 6.2. A fuzzy set $A$ in $E$ is fuzzily ortho-convex if for all $x \in E, y \in E$, such that $\langle x, y\rangle$ is either horizontal or vertical, and all point $z \in\langle x, y\rangle$ the inequality $\chi_{A}(z) \geq \min \left\{\chi_{A}(x), \chi_{A}(y)\right\}$ holds.

We take $X=E, Y=[0,1] \times E \times E, S=2^{E}$,

$$
\mathcal{S}=\left\{s_{x, y} \mid x \in E, y \in E,\langle x, y\rangle \text { is either horizontal or vertical }\right\},
$$

where $s_{x, y}: S \rightarrow 2^{Y}$ is defined by $s_{x, y}=\left\{\left(\chi_{A}(z), x, y\right) \mid z \in\langle x, y\rangle\right\}, \mathcal{R}=\left\{r_{u, v} \mid u \in\right.$ $E, v \in E\}, r_{u, v}: 2^{E} \rightarrow 2^{Y}$,

$$
r_{u, v}(D)=\left\{(t, u, v) \mid t \in\left[\min \left\{\chi_{D}(z) \mid z \in\langle x, y\rangle\right\}, \max \left\{\chi_{D}(u), \chi_{D}(v)\right\}\right]\right\} .
$$


Theorem 6.1. A set $A \subseteq E$ is fuzzily ortho-convex in $E$ if and only if $A$ is special partially $(a, e)-((S, \mathcal{S}), \mathcal{R})$ convex.

A directional construction as in Example 4.4 leads to a particular discrete case. Applications in pattern recognition are described in [6].

Example 6.2. Fuzzy ortho-concavity [7].

Let $E$ be the Euclidean plane.

Definition 6.3. A fuzzy set $A$ in $E$ is fuzzily ortho-concave if for all $x \in E, y \in E$, which are either on a horizontal line or on a vertical one, and each $z \in\langle x, y\rangle$ the inequality $\chi_{A}(z) \leq \max \left\{\chi_{A}(x), \chi_{A}(y)\right\}$ occurs.

We take $X=E, Y=[0,1] \times E \times E, S=2^{E}, \mathcal{S}=\left\{s_{x, y} \mid x \in E, \quad y \in\right.$ $E,\langle x, y\rangle$ is either horizontal or vertical $\}$, where $s_{x, y}: S \rightarrow 2^{Y}$ is defined by $s_{x, y}=$ $\left\{\left(\chi_{A}(z), x, y\right) \mid z \in\langle x, y\rangle\right\}, \mathcal{R}=\left\{r_{u, v} \mid u \in E, v \in E\right\}, r_{u, v}: 2^{E} \rightarrow 2^{Y}$,

$$
r_{u, v}(D)=\left\{(t, u, v) \mid t \in\left[\min \left\{\chi_{D}(z) \mid z \in\langle x, y\rangle\right\}, \max \left\{\chi_{D}(u), \chi_{D}(v)\right\}\right]\right\} .
$$

Theorem 6.2. A set $A \subseteq E$ is fuzzily ortho-concave in $E$ if and only if $A$ is special partially $(a, e)-((S, \mathcal{S}), \mathcal{R})$ convex.

It is obvious that the $((S, \mathcal{S}), \mathcal{R})$ terminology allows us to treat this concavity as a convexity with discrete particular cases.

This classification of discrete convexity concepts offers more tools to construct discrete theories that are approximations of existing continuous versions. Papers [13]-[16] and [28]-[31] contain more possibilities of creating framework for both theoretical and practical applications of various types of convexity properties that contain discrete particular cases.

In this paper we did not consider the axiomatic convexities that allow the construction of various discrete models, in lattices or in various ordered sets. They provide the reader with other methods to build discrete convexity theories. Here we preferred to make a unifying systematic discussion of the discrete convexities that appear in various applications in integer programming, rational programming or in pattern recognition.

\section{References}

1. L. Blaga and L. Lupşa, Convexity space with respect to a given set, in Generalized Convexity, Generalized Monotonicity, J. P. Crouzeix, J. E. Martinez-Legaz and M. Volle (eds.), pp. 199-208, Kluwer, Dordrecht, 1998.

2. J. M. Chassery, Convexité et enveloppe convexe dans $\mathbf{Z}^{2}$, Séminaire Analyse Numérique, No. 297, IMAG, Grenoble, 1978.

3. J. M. Chassery, Discrete convexity: definition, parametrization and comparability with continuous convexity, Comput. Vision Graphics Image Process. 21 (1983), 326-344.

4. J. M. Chassery, Reprézentation discrète, interprétation numérique et description des images: des concepts à l' applications, Thèse, Université Scientifique et Médicale de Grénoble, 1984. 
5. B. B. Chaudhuri, Some shape definitions in fuzzy geometry of space, Pattern Recognition Lett. 12 (1991), 531-535.

6. B. B. Chaudhuri, Fuzzy convex hull determination in 2D-space, Pattern Recognition Lett. 12 (1991), 591-594.

7. B. B. Chaudhuri, Concave fuzzy set: a concept complementary to the convex fuzzy set, Pattern Recognition Lett. 13 (1992), 103-106.

8. G. Cristescu, Convexity close by undetectable concavity, in Proc. Sci. Com. Meeting of the Aurel Vlaicu University, Third Edition, Arad, 16-17 May 1996, 14A, pp. 45-50, Aurel Vlaicu University, Arad, 1996.

9. G. Cristescu, Convexities with respect to a set and two behaviours, in Proc. Sci. Com. Meeting of the Aurel Vlaicu University, Third Edition, Arad, 16-17 May 1996, 14A, pp. 35-43, Aurel Vlaicu University, Arad, 1996.

10. G. Cristescu, Sets looking convex-like, in Proc. $5^{\text {th }}$ Symposium on Descriptive Geometry, Design, Engineering and Computer Graphics, "Graphics, a scientific language”, 17-19 ${ }^{\text {th }}$ June 1996, pp. 725-730, Politehnica University, Timişoara, 1996.

11. G. Cristescu, On the approximation of the classical convexity, in Proc. Sci. Com. Meeting of the Aurel Vlaicu University, Fourth Edition, Arad, 29-30 October 1997, pp. 39-46, Aurel Vlaicu University, Arad, 1997.

12. G. Cristescu, Convexity related to the coarseness of the concavity, Rév. Anal. Numer. Theory Approx., 26(1-2) (1997), 29-37.

13. G. Cristescu, Approximation of the classical convexity in totally bounded metric spaces, Bull. Appl. Comput. Math., LXXXV-B (1998), 191-196.

14. G. Cristescu, Weak convergence of some convexities to the classical convexity, in Academia Tehnică Militară, A XXVIII-a Sesiune de Comunicări ştiinţifice cu Participare Internaţională organizată cu ocazia Sărbătoririi a 50 de Ani de la Înfiinţarea Academiei Tehnice Militare, Secţiunea Matematică şi Fizică, Subsecţiunea 1 Matematică, Bucureşti, 21-22 Octombrie 1999, pp. 86-91, Military Technical Academy, Bucharest, 1999.

15. G. Cristescu, Behaviours: Implications and Applications in Convexity Recognition and in Topology, Monographical Booklets in Applied and Computer Mathematics, MB-14, Technical University of Budapest, Budapest, 2000.

16. G. Cristescu and L. Lupşa, Non-Connected Convexities and Applications, Kluwer, Dordrecht, 2002.

17. L. Janos and A. Rosenfeld, Some results on fuzzy (digital) convexity, Pattern Recognition 15(5) (1982), 379-382.

18. C. E. Kim, On the cellular convexity of complexes, IEEE Trans. Pattern Anal. Mach. Intel. 3 (1981), 617-625.

19. C. E. Kim and A. Rosenfeld, On the convexity of digital regions, in Proc. $5^{\text {th }} J C P R$, pp. $1010-1015,1980$.

20. C. E. Kim and J. Sklansky, Digital and cellular convexity, Pattern Recognition 15(5) (1982), 359-367.

21. L. Latecki, A. Rosenfeld and R. Silverman, Generalized convexity: $\mathrm{CP}_{3}$ and boundaries of convex sets, Pattern Recognition 28(8) (1995), 1911-1999.

22. L. Lupşa, On the structure of the essentially efficient solutions of an integer vectorial programming problem (in Romanian), in Seminarul itinerant de ecuaţii funcţionale, aproximare şi convexitate, 1980, 16-17 mai; Cluj-Napoca, pp. 77-89, Babeş-Bolyai University, Cluj-Napoca, 1980.

23. L. Lupşa, On a convexity notion (in Romanian), in Seminarul itinerant de ecuatii functionale, aproximare şi convexitate, 1980, 7-8 noiembrie; Timişoara, pp. 127-135, University of Timişoara, Timişoara, 1980.

24. L. Lupşa, On the strongly convex sets with respect to a given set (in Romanian), in Seminarul itinerant de ecuaţii funcţionale, aproximare şi convexitate, 1981, mai; Cluj-Napoca, pp. 217-227, Babeş-Bolyai University, Cluj-Napoca, 1981.

25. L. Lupşa, Separation of strong convex sets, in Proc. Itinerant Seminar on Functional Equations, Approximation and Convexity, 1983, Cluj-Napoca, pp. 85-86, Babeş-Bolyai University, Cluj-Napoca, 1983.

26. L. Lupşa, Slack convexity with respect to a given set, in Proc. Itinerant Seminar on Functional Equations, Approximation and Convexity, 1985, Cluj-Napoca, pp. 107-114, Babeş-Bolyai University, Cluj-Napoca, 1985.

27. L. Lupşa, Some types of convexity with respect to a given set, in Seminar on Optimization Theory, Babeş-Bolyai University, Cluj-Napoca, 1986, pp. 89-114.

28. L. Lupşa, On integer convex sets, I, in Proc. Itinerant Seminar on Functional Equations, Approximation and Convexity, 1988, Cluj-Napoca, pp. 205-208, Babeş-Bolyai University, Cluj-Napoca, 1988. 
29. L. Lupşa, On integer convex sets, II, in Proc. Itinerant Seminar on Functional Equations, Approximation and Convexity, 1989, Cluj-Napoca, pp. 211-216, Babeș-Bolyai University, Cluj-Napoca, 1989.

30. L. Lupşa, Induced convexity (in Romanian), in Seminarul itinerant "Tiberiu Popoviciu" de ecuaţii funçionale, aproximare şi convexitate, 16 mai-21 mai 1995, Cluj-Napoca, pp. 61-64, Babeş-Bolyai University, Cluj-Napoca, 1995.

31. L. Lupşa, L. Blaga, D. Duca and E. Duca, Some types of $(g, h, M)$-convexity, in Séminaire de la théorie de la meilleure approximation, convexité et optimisation, pp. 161-170, SRIMA, Cluj-Napoca, 2000.

32. M. Minsky and S. Papert, Perceptrons, MIT Press, Cambridge, MA, 1969.

33. G. J. E. Rawlins and D. Wood, Ortho-convexity and its generalizations, in Computational Morphology, pp. 137-152, Elsevier, Amsterdam, 1988.

34. A. Rosenfeld and A Kak, Digital Picture Processing, Academic Press, New York, 1976.

35. A. Rosenfeld and A Kak, Digital Picture Processing, Second Edition, Vol. 1-2, Academic Press, San Diego, CA, 1982.

36. J. Sklansky, Recognition of convex blobs, Pattern Recognition 2 (1970), 3-10.

37. F. A. Valentine, A three point convexity property, Pacific J. Math. 7 (1957), 1227-1235.

38. P. L. Yu, Cone convexity, cone extreme points and nondominated solutions in decision problems with multiobjectives, J. Optim. Theory Appl. 14(3) (1974), 319-377.

Received May 15, 2002, and in revised form July 18, 2003. Online publication February 18, 2004. 